\title{
Interference in spectrum of radiation from doubly scattered charged particle
}

\author{
M.V. Bondarence* and N.F. Shul'ga \\ NSC Kharkov Institute of Physics and Technology, \\ 1 Academic Street, 61108 Kharkov, Ukraine and \\ V.N. Karazine Kharkov National University, 4 Svobody Square, 6107r Kharkov, Ukraine
}

(Dated: July 9, 2018)

\begin{abstract}
Existence of different types of interference in the spectrum of radiation emitted by a doubly hard scattered electron is demonstrated. The spectrum develops oscillations in two regions: the hard, where the oscillations depend on the electron Lorentz factor, and the soft, where the oscillations depend on the electron scattering angles. This interference pattern owes to the presence of jetlike radiation configurations, formed by a piecewise-rectilinearly moving electron and the accompanying photon. The corresponding nondipole decomposition relation is derived. Notions describing proper field formation and interference, and presumably being applicable more generally, are discussed in detail.

PACS numbers: 41.60.-m, 13.87.-a
\end{abstract}

\section{INTRODUCTION}

Relatively recently, it was recognized that gamma radiation from ultrarelativistic electrons in noncrystalline finite targets can exhibit salient "nondipole" interference effects when the electron deflection angles exceed the natural scale for radiation emission angles set by the electron inverse Lorentz factor [1-4]. The existence of such effects was confirmed by CERN experiments, which observed oscillations in the hard part of angle-integral photon emission spectra from $\sim 200 \mathrm{GeV}$ electrons passing through a pair of amorphous foils separated by a submillimeter gap [5]. Understanding of the behavior of radiation spectra in such cases, however, still seems to be incomplete. Reasoning of 4 had explained the shape and location of features in the hard spectral domain, whereas the soft domain was assumed to be featureless. But that holds only for spectra averaged over a broad scattering angle distribution like that in amorphous foils in experiments [5].

Qualitative assessment of any interference effects in radiation relies on the notion of photon formation length, which is confronted with intrinsic geometrical scale(s) in the problem (in the case mentioned above - with the interfoil distance). The conventionally defined photon formation length

$$
l_{\mathrm{f}}=\frac{2}{\omega\left(\gamma^{-2}+\theta^{2}\right)},
$$

besides the photon frequency $\omega$, depends on its emission angles $\theta$, and through them, indirectly, on electron scattering angles. Thus, in practice, one has first to accurately determine which angles are relevant, and from which particular direction they are to be counted off. For instance, the Landau-Pomeranchuk-Migdal suppression of the soft part of radiation spectrum from an

*Electronic address: bon@kipt.kharkov.ua electron in a thick amorphous target [6, 7] is known to be described by the photon formation length depending on the mean square deflection angle accumulated by the electron in the target, with $\theta^{2} \sim\left\langle\chi^{2}\right\rangle \gg \gamma^{-2}$ [8 10 . On the contrary, the aforementioned case of nondipole bremsstrahlung on a pair of amorphous foils [5] seems to be related only with the free photon formation length (defined in the absence of electron scattering), when $\theta \lesssim \gamma^{-1}$ 4. It may be puzzling how to reconcile this with the fact that the corresponding oscillations fully develop only when typical scattering angles overtake the inverse Lorentz factor. Basically, that can be due to incidental insensitivity of period and phase of the oscillations in the hard spectral domain to the mean square angles of scattering in both targets, but a principal question remains: Is there a signature of the electron scattering angle dependent photon formation length anywhere among the radiation observables?

To answer this question, and pave the way for further developments, it is expedient to revisit the cornerstone problem - radiation from an electron undergoing successive double scattering through certain angles, not subject to any averaging. Such a problem was discussed in a number of instances before: The space-time evolution of the retarded electromagnetic field was analyzed by Purcell [11, whereas general properties of the quantum amplitude, by Feynberg [12].

The object of our study, however, will be the radiation spectrum integrated over emission angles, to which experimental observation in the ultrarelativistic case is usually restricted, as long as photons are typically emitted in a narrow cone around the forward direction. For this observable, the interference pattern appears to be richer than one might naively expect, and effectively involves manifestations of several photon formation lengths, showing up in different spectral regions. We will deduce the corresponding decomposition relation, and investigate the physical meaning of its entries.

Examination of the emerging structure then leads us to more profound conclusions. All the discovered spec- 
tral features prove to be consequences of the presence of electron-photon jetlike configurations, which can participate in interference phenomena in spite of their narrow collimation properties. That gives rise to notions such as intermediate electron line and "radio" contributions (among which the first is independent of electron deflection angles, while the second is independent of the electron Lorentz factor), and "proper field form factors" multiplying the radio contribution. The latter notions may be applicable in a broad class of problems. To reach their versatile understanding, it appears beneficial to discuss the problem from several points of view.

Specifically, after setting forth the initial assumptions in Sec. II, we turn in Sec. III to evaluating the spectrum in terms of photon emission angles. That reveals the existence of jetlike and interjet radiation, but hides their spatial ordering. Additional insight is gained in Sec. IV by considering the process in transverse coordinates (impact parameters), which best elucidates the origin of electron proper field form factors. In Sec. V that is complemented by a study of longitudinal evolution of photon formation and interference, aiming to demonstrate that the long and short scales anticipated to be photon formation lengths do correspond to the process development in time. Section VI includes a brief analysis of experimental realizability of the considered process. Section VII provides the summary. In the Appendix, we derive the covariant form of the double time integral representation for the radiation spectrum, and highlight its gauge properties, which prove relevant in the present context.

\section{PRELIMINARIES}

For the interference effects discussed in this paper to be pronounced, energies of the emitted photons are to be low compared with the electron energy. That creates premises for applicability of classical electrodynamics: The possible quantal nature of the electron motion in the domains of scattering is inessential provided the photon formation length greatly exceeds the extent of each of those scattering areas. Then, the factorization theorem asserts that the differential probability of the entire bremsstrahlung process splits into a product of two differential cross sections of elastic electron scattering and the differential probability of emission of an electromagnetic wave from an angle-shaped charged particle trajectory [9. 13. Our study in this paper will focus on the photon emission probability alone, which is tractable purely classically. Moreover, at high energies, the motion of the electron in macroscopic-field deflectors may be semiclassical, as well (see Sec. VI).

We thus consider radiation from a classical charge $e$ (physically representing an electron or positron) moving along a double-angle-shaped trajectory $\boldsymbol{r}(t)$, with velocity $\boldsymbol{v}(t)=d \boldsymbol{r} / d t$ depending on time $t$. Specifically, as was mentioned in the Introduction, we shall evaluate the angle-integral radiation spectrum, which in the ultrarel- ativistic case $\gamma=\left(1-v^{2}\right)^{-1 / 2} \ggg 1$ is the prime experimental observable. It involves several operations [14]: a time integral with conjugate plane-wave factor $e^{i \omega t-i \boldsymbol{k} \cdot \boldsymbol{r}}$ depending on the photon frequency $\omega$ and emission direction $\boldsymbol{n}=\boldsymbol{k} / \omega$, subsequent amplitude squaring, and integration over directions of $\boldsymbol{n}$ :

$$
\frac{d I}{d \omega}=\omega^{2} \int d^{2} n\left|\frac{e}{2 \pi} \int_{-\infty}^{\infty} d t[\boldsymbol{n} \times \boldsymbol{v}(t)] e^{i \omega t-i \boldsymbol{k} \cdot \boldsymbol{r}(t)}\right|^{2} .
$$

To reach proper understanding of its behavior, it is desirable to reduce (2) at least to a single integral, enabling clear-cut isolation of dominant contributions, and thereby, a rigorous measure of the radiation coherence. The physical meaning of the latter contributions will depend on the nature of the last integration variable. We will describe three most informative approaches, and demonstrate that although, inevitably, they all lead to the same structure of the final result, their interpretations elucidate different aspects of the radiation process, thus being mutually complementary. Their amalgamation then leads to a cogent picture for interference effects in bremsstrahlung at electron rescattering, which may also prove relevant for in other highly nondipole radiation problems.

\section{ANALYSIS IN TERMS OF ANGULAR DISTRIBUTIONS}

To fully describe the electron trajectory, we denote its successive elastic deflection angles as $\chi_{1}$ and $\chi_{2}\left[\gamma^{-1} \ll\right.$ $\left.\chi_{1,2} \ll 1\right]$, and the separation time (or length) ${ }^{1}$ between the scatterings as $T$ (see Fig. 1). Noncoplanarity of the scattering angles is characterized by the azimuthal angle between them, $\varphi_{12}=\arccos \left(\chi_{1} \cdot \chi_{2} /\left|\chi_{1}\right|\left|\chi_{2}\right|\right)$. In this abrupt scattering case, it is straightforward to integrate in (2) first over time. That gives

$$
\begin{aligned}
& \frac{d I}{d \omega}=\frac{e^{2}}{\pi^{2}} \int d^{2} \theta\left\{\left[\frac{\boldsymbol{\theta}}{\gamma^{-2}+\theta^{2}}-\frac{\boldsymbol{\theta}+\boldsymbol{\chi}_{1}}{\gamma^{-2}+\left(\boldsymbol{\theta}+\chi_{1}\right)^{2}}\right]^{2}\right. \\
& +\left[\frac{\boldsymbol{\theta}-\boldsymbol{\chi}_{2}}{\gamma^{-2}+\left(\boldsymbol{\theta}-\boldsymbol{\chi}_{2}\right)^{2}}-\frac{\boldsymbol{\theta}}{\gamma^{-2}+\theta^{2}}\right]^{2} \\
& +2\left[\frac{\boldsymbol{\theta}}{\gamma^{-2}+\theta^{2}}-\frac{\boldsymbol{\theta}+\boldsymbol{\chi}_{1}}{\gamma^{-2}+\left(\boldsymbol{\theta}+\chi_{1}\right)^{2}}\right] \\
& \left.\cdot\left[\frac{\boldsymbol{\theta}-\chi_{2}}{\gamma^{-2}+\left(\boldsymbol{\theta}-\chi_{2}\right)^{2}}-\frac{\boldsymbol{\theta}}{\gamma^{-2}+\theta^{2}}\right] \cos \frac{\omega T}{2 \gamma^{2}}\left(1+\gamma^{2} \theta^{2}\right)\right\} .(3)
\end{aligned}
$$

The behavior of the integrand of Eq. 3 in the $\boldsymbol{\theta}$ plane (the angular distribution of radiation) is depicted in Figs. 2 and 3 for a case of exemplary scattering angles $\left|\chi_{1}\right| \sim\left|\chi_{2}\right| \sim 30 \gamma^{-1}$, and progressively increasing values

\footnotetext{
${ }^{1}$ We adopt units in which the speed of light equals unity.
} 


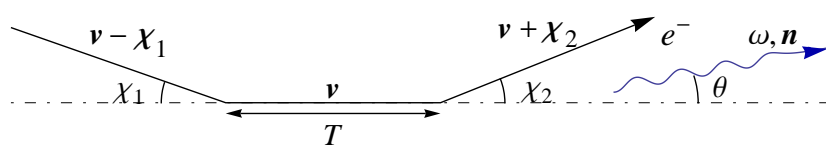

FIG. 1: Geometry of the considered electron scattering process and the accompanying radiation. For symmetry's sake, all the angles are counted off from the intermediate electron velocity.

of $\frac{\omega T}{2 \gamma^{2}}$. One can visualize there three cones of radiation (associated with one internal and two external electron lines), with concentric interference rings about the internal line. The outreach of the latter rings depends on $\omega T$. At $\omega T \chi^{2} / 2 \rightarrow 0$, the rings expand to infinity, and the angular distribution of radiation at finite $\theta$ [the inner part of Fig. 2(a)] tends to that at single electron scattering through angle $\chi_{1}+\chi_{2}$ (cf., e.g., [16]). ${ }^{2}$ At some finite $\omega$, the radius of the rings starts to come close to one of the deflection angles. Successively, when this radius by far exceeds $\chi_{1}, \chi_{2}$, there exists only interference between the external lines [Fig. 2(a)], when it becomes on a par with the size of (one of the) $\chi$ 's, there emerges interference between the internal and an external line [Fig. 2(b)], and when it falls below $\chi_{1}, \chi_{2}$, the latter interference is lost, as well [Fig. 2(c)], and only interference within the internal line survives (Fig. 33). ${ }^{3}$

For arbitrary $\chi_{1}$ and $\chi_{2}$, the $\omega$-independent (noninterference) part of (3) consists of two separate ("BetheHeitler") contributions from the scattering vertices, each of which is given by the well-known bremsstrahlung formula

$$
\begin{aligned}
\frac{d I_{\mathrm{BH}}}{d \omega}(\gamma \chi) & =\frac{e^{2}}{\pi^{2}} \int d^{2} \theta\left[\frac{\boldsymbol{\theta}-\boldsymbol{\chi}}{\gamma^{-2}+(\boldsymbol{\theta}-\chi)^{2}}-\frac{\boldsymbol{\theta}}{\gamma^{-2}+\theta^{2}}\right]^{2} \\
\underset{\chi \gg \gamma^{-1}}{\simeq} \frac{2 e^{2}}{\pi}\left(\ln \gamma^{2} \chi^{2}-1\right) . & (4 \mathrm{~b})
\end{aligned}
$$

Integral 4a converges due to mutual cancellation between the terms in the brackets.

In contrast, for the nontrivial, interference part of (3), the terms in its integrand may be treated independently, because there the convergence is provided by the cosine factor. The key observation is that different terms in the integrand give dependence of $d I / d \omega$ on $\omega$ at different scales. Specifically, separating $\chi$-dependent and

\footnotetext{
${ }^{2}$ It should be mentioned that Fig. 1 in [16, corresponding to the total deflection angle $10 \gamma^{-1}$, did not belong to the ultranondipole regime yet, whereas our figure with $\left|\chi_{1}+\chi_{2}\right| \sim 50 \gamma^{-1}$ does. That is responsible for residual differences between the plots.

3 Angular distributions similar to those in Fig. 3 were discovered previously in other but related physical problems: radiation from an electron in a straight section between magnets in a storage ring, or in more elaborate synchrotron radiation sources (see 17 [18] and references therein).
}

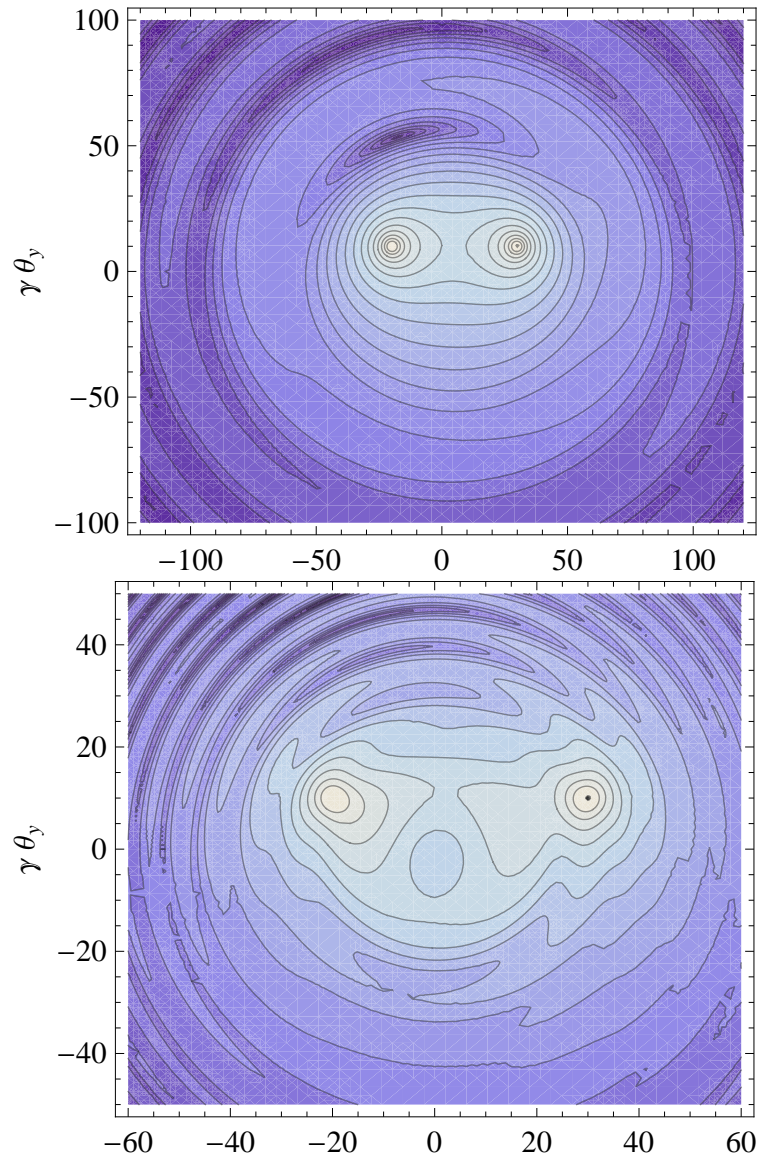

(a)



(b)

(c)

FIG. 2: Angular distributions of radiation from a doubly scattered electron. Directions of initial and final electron motion coincide with centers of the leftmost and the rightmost jetlike features. (a) $\frac{\omega T}{2 \gamma^{2}}=0.001$, (b) $\frac{\omega T}{2 \gamma^{2}}=0.01$, (c) $\frac{\omega T}{2 \gamma^{2}}=0.1$. For higher $\omega$, see Fig. 3 below. For discussion see text. 


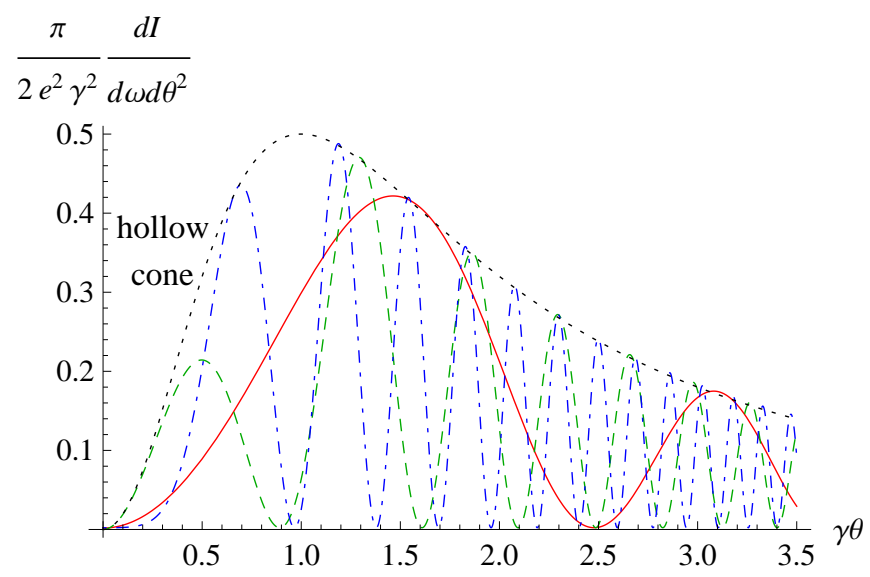

FIG. 3: Angular distribution of radiation intensity around the direction of the intermediate electron velocity, for $\chi_{1}=$ $\chi_{2}=30 \gamma^{-1}$ (there is no sensitivity to those angles, provided they are large enough), and different $\omega$ in the hard region. In the displayed domain, the distribution is virtually axially symmetric and $\chi$-independent. Red solid curve, $\frac{\omega T}{2 \gamma^{2}}=0.9$ (the main maximum of $d I / d \omega$ in the hard region). Green dashed, $\frac{\omega T}{2 \gamma^{2}}=3.5$ (the following minimum of $d I / d \omega$ ). Blue dot-dashed, $\frac{\omega T}{2 \gamma^{2}}=6.5$ (the secondary maximum of $d I / d \omega$ ). For the corresponding features in $d I / d \omega$, cf. Fig. 4(a) below. Black dotted curve, the envelope $\frac{2 \gamma^{2} \theta^{2}}{\left(1+\gamma^{2} \theta^{2}\right)^{2}}$.

-independent parts gives

$$
\begin{aligned}
\frac{d I}{d \omega}= & \frac{d I_{\mathrm{BH}}}{d \omega}\left(\gamma \chi_{1}\right)+\frac{d I_{\mathrm{BH}}}{d \omega}\left(\gamma \chi_{2}\right) \\
& +\frac{2 e^{2}}{\pi}\left[g\left(\frac{\omega T}{2 \gamma^{2}}\right)+r\left(\chi_{1}, \chi_{2}, \gamma^{-1}, \omega T\right)\right],
\end{aligned}
$$

where $^{4}$

$$
\begin{aligned}
g\left(\frac{\omega T}{2 \gamma^{2}}\right) & =-\frac{1}{\pi} \int d^{2} \theta \frac{\theta^{2}}{\left(\gamma^{-2}+\theta^{2}\right)^{2}} \cos \frac{\omega T}{2 \gamma^{2}}\left(1+\gamma^{2} \theta^{2}\right) \\
& =\operatorname{Ci}\left(\frac{\omega T}{2 \gamma^{2}}\right)+\frac{\omega T}{2 \gamma^{2}} \operatorname{si}\left(\frac{\omega T}{2 \gamma^{2}}\right)+\cos \frac{\omega T}{2 \gamma^{2}},(6 \mathrm{~b})
\end{aligned}
$$

with $\operatorname{Ci}(z)=-\int_{z}^{\infty} \frac{d x}{x} \cos x, \operatorname{si}(z)=-\int_{z}^{\infty} \frac{d x}{x} \sin x$ the

\footnotetext{
${ }^{4}$ In [4], function $g\left(\frac{\omega T}{2 \gamma^{2}}\right)$ was denoted as $g_{11}\left(0, \frac{\omega T}{2 \gamma^{2}}\right)$, to distinguish it from dipole or mixed-strength radiation cases. Here, confining ourselves to the strongly nondipole case alone, we omit noninformative labels.
}

integral cosine and sine functions 20, and

$$
\begin{array}{r}
r\left(\boldsymbol{\chi}_{1}, \boldsymbol{\chi}_{2}, \gamma^{-1}, \omega T\right)=\frac{1}{\pi} \int d^{2} \theta \cos \frac{\omega T}{2 \gamma^{2}}\left(1+\gamma^{2} \theta^{2}\right) \\
\times\left\{\frac{\boldsymbol{\theta}}{\gamma^{-2}+\theta^{2}} \cdot\left[\frac{\boldsymbol{\theta}+\boldsymbol{\chi}_{1}}{\gamma^{-2}+\left(\boldsymbol{\theta}+\boldsymbol{\chi}_{1}\right)^{2}}+\frac{\boldsymbol{\theta}-\boldsymbol{\chi}_{2}}{\gamma^{-2}+\left(\boldsymbol{\theta}-\boldsymbol{\chi}_{2}\right)^{2}}\right]\right. \\
\left.-\frac{\boldsymbol{\theta}+\boldsymbol{\chi}_{1}}{\gamma^{-2}+\left(\boldsymbol{\theta}+\boldsymbol{\chi}_{1}\right)^{2}} \cdot \frac{\boldsymbol{\theta}-\boldsymbol{\chi}_{2}}{\gamma^{-2}+\left(\boldsymbol{\theta}-\boldsymbol{\chi}_{2}\right)^{2}}\right\}
\end{array}
$$

Note that $\int_{0}^{\infty} d \omega g\left(\frac{\omega T}{2 \gamma^{2}}\right)=\int_{0}^{\infty} d \omega r\left(\chi_{1}, \chi_{2}, \gamma^{-1}, \omega T\right)=$ 0 , as a consequence of locality of electromagnetic energy emission in classical electrodynamics (see, e.g., 4]).

At $\omega T / 2 \gamma^{2} \gtrsim 1$, when typical contributing angles are restricted by $\theta \lesssim \gamma^{-1} \ll \chi$, part $r$ is suppressed compared to $g$ by inverse powers of $\gamma \chi$, and can be neglected. Therewith,

$$
\frac{d I}{d \omega} \underset{\frac{\omega \bar{T}}{2 \gamma^{2}} \gtrsim 1}{\simeq} \frac{d I_{\mathrm{BH}}}{d \omega}\left(\gamma \chi_{1}\right)+\frac{d I_{\mathrm{BH}}}{d \omega}\left(\gamma \chi_{2}\right)+\frac{2 e^{2}}{\pi} g\left(\frac{\omega T}{2 \gamma^{2}}\right) .
$$

In the formal limit $\omega T / 2 \gamma^{2} \rightarrow \infty$, the spectrum exhibits decreasing harmonic oscillations $[4]^{5}$

$$
\frac{d I}{d \omega} \underset{\frac{\omega T}{2 \gamma^{2}} \gg 1}{\simeq} \frac{d I_{\mathrm{BH}}}{d \omega}\left(\gamma \chi_{1}\right)+\frac{d I_{\mathrm{BH}}}{d \omega}\left(\gamma \chi_{2}\right)+\frac{2 e^{2}}{\pi}\left(\frac{2 \gamma^{2}}{\omega T}\right)^{2} \cos \frac{T}{l_{0}(\omega)},
$$

where

$$
l_{0}(\omega)=\frac{2 \gamma^{2}}{\omega}
$$

stands for the "free" photon formation length. It is relevant here insofar as between the hard scatterings the electron moves strictly rectilinearly (should there be some medium or external field along its path, the situation might drastically change). Note, too, that the decrease here follows the law $\sim \omega^{-2}$ instead of $\sim \omega^{-1}$, owing to the integrand in 6 a) vanishing at $\theta \rightarrow 0$ (a "hollow cone" distribution of radiation emitted from an isolated straight electron line), due to the vector and gauge character of electromagnetic radiation.

When $\omega \rightarrow 0$, function (6b) logarithmically diverges, so, ultimately, approximation (8) must break down. That reflects physical limitedness of separate treatment of individual terms in Eq. (5). ${ }^{6}$ At sufficiently low $\omega$, all the

\footnotetext{
${ }^{5}$ Strictly speaking, 9 becomes numerically accurate only in a rather far asymptotic region (see [4]). To reach higher precision, it may be worth retaining the next-to-leading order term in the phase, but we shall not indulge into such complications in the present paper.

${ }^{6}$ It must be remembered that the interpretation of individual terms in 2 as stemming from isolated parts of the electron's trajectory is not gauge invariant, because at the ends of a finite trajectory segment there is no conservation of charge. In this
} 
terms in (5) become comparable and simultaneously important. Nonetheless, there still remains room for simplifications: At $\chi \gg \gamma^{-1}$, it is justified to entirely neglect terms containing $\gamma^{-2}$ (provided $\left|\chi_{1}+\chi_{2}\right| \gg \gamma^{-1}$, to avoid a case of overlap of singularities, which will be touched upon later). Physically, that means that in the softest spectral (here called radio) region, the ensemble of segments of the electron's trajectory acts like a single antenna (cf., e.g., 19] - viz., like a long "wire", which is significantly deformed within the photon formation length, so that the electric current along it, representing the passing electron, may be regarded as flowing exactly at the speed of light. Evaluation of the corresponding integral gives $^{7}$

$$
\begin{aligned}
& r\left(\boldsymbol{\chi}_{1}, \boldsymbol{\chi}_{2}, 0, \omega T\right) \\
& =\frac{1}{\pi} \int d^{2} \theta\left\{\frac{\boldsymbol{\theta}}{\theta^{2}} \cdot\left[\frac{\boldsymbol{\theta}+\chi_{1}}{\left(\boldsymbol{\theta}+\chi_{1}\right)^{2}}+\frac{\boldsymbol{\theta}-\chi_{2}}{\left(\boldsymbol{\theta}-\chi_{2}\right)^{2}}\right]\right. \\
& \left.-\frac{\boldsymbol{\theta}+\chi_{1}}{\left(\boldsymbol{\theta}+\chi_{1}\right)^{2}} \cdot \frac{\boldsymbol{\theta}-\chi_{2}}{\left(\boldsymbol{\theta}-\chi_{2}\right)^{2}}\right\} \cos \frac{\omega T \theta^{2}}{2} \\
& =\int_{\chi_{1}^{2}}^{\infty} \frac{d \theta^{2}}{\theta^{2}} \cos \frac{\omega T \theta^{2}}{2}+\int_{\chi_{2}^{2}}^{\infty} \frac{d \theta^{2}}{\theta^{2}} \cos \frac{\omega T \theta^{2}}{2} \\
& -\int_{\chi_{1}^{2}}^{\infty} d \theta^{2} \cos \frac{\omega T \theta^{2}}{2} \mathfrak{R e} \frac{1}{\chi_{1} \bar{\chi}_{2}+\theta^{2}} \\
& -\int_{\chi_{2}^{2}}^{\infty} d \theta^{2} \cos \frac{\omega T \theta^{2}}{2} \mathfrak{R e} \frac{1}{\chi_{1} \bar{\chi}_{2}+\theta^{2}} \\
& +\int_{0}^{\infty} d \theta^{2} \cos \frac{\omega T \theta^{2}}{2} \mathfrak{R e} \frac{1}{\chi_{1} \bar{\chi}_{2}+\theta^{2}},
\end{aligned}
$$

where $\chi_{1} \bar{\chi}_{2}=\left(\chi_{1 x}+i \chi_{1 y}\right)\left(\chi_{2 x}-i \chi_{2 y}\right)=\left|\chi_{1}\right|\left|\chi_{2}\right| e^{i \varphi_{12}}$. Representation (13a) in terms of single integrals is already suitable for assessment of coherence effects, but

and the following sections, we work, specifically, in the radiative gauge. Nevertheless, since the entire spectral-angular distribution (3) is gauge invariant, its terms with different dependencies on $\chi_{1}, \chi_{2}$ may be singled out at least formally, and treated separately in this sense.

${ }^{7}$ Integration over the azimuth of $\boldsymbol{\theta}$ is alleviated by introducing a complex variable $\zeta=\theta_{x}+i \theta_{y}$ for Cartesian components $\theta_{x}, \theta_{y}$ of vector $\boldsymbol{\theta}$, and evaluating the encountered integrals by residues:

$$
\begin{aligned}
\int d \phi_{\boldsymbol{\theta}} \frac{\boldsymbol{\theta} \cdot(\boldsymbol{\theta}-\boldsymbol{\chi})}{(\boldsymbol{\theta}-\boldsymbol{\chi})^{2}} & =\mathfrak{R e} \int d \phi_{\boldsymbol{\theta}} \frac{\left(\theta_{x}+i \theta_{y}\right)\left(\theta_{x}-i \theta_{y}-\chi\right)}{\left(\theta_{x}+i \theta_{y}-\chi\right)\left(\theta_{x}-i \theta_{y}-\chi\right)} \\
& =\mathfrak{R e} \frac{1}{i} \oint_{|\zeta|=|\boldsymbol{\theta}|} \frac{d \zeta}{\zeta-\chi}=2 \pi \vartheta(|\boldsymbol{\theta}|-|\boldsymbol{\chi}|),
\end{aligned}
$$

with $\vartheta(\ldots)$ the Heaviside unit step function, and

$$
\begin{array}{r}
\int d \phi_{\boldsymbol{\theta}} \frac{\left(\boldsymbol{\theta}+\chi_{1}\right) \cdot\left(\boldsymbol{\theta}-\chi_{2}\right)}{\left(\boldsymbol{\theta}+\chi_{1}\right)^{2}\left(\boldsymbol{\theta}-\chi_{2}\right)^{2}}=\mathfrak{R e} \oint_{|\zeta|=|\boldsymbol{\theta}|} d \phi_{\zeta} \frac{1}{\left(\bar{\zeta}-\bar{\chi}_{2}\right)\left(\zeta+\chi_{1}\right)} \\
=\mathfrak{R e} \frac{1}{\bar{\chi}_{2} \chi_{1}+|\zeta|^{2}} \oint_{|\zeta|=|\boldsymbol{\theta}|} d \phi_{\zeta}\left[\frac{\bar{\zeta}}{\bar{\zeta}-\bar{\chi}_{2}}-\frac{\chi_{1}}{\zeta+\chi_{1}}\right] \\
=2 \pi\left[\vartheta\left(|\boldsymbol{\theta}|-\left|\chi_{2}\right|\right)-\vartheta\left(\left|\chi_{1}\right|-|\boldsymbol{\theta}|\right)\right] \mathfrak{R e} \frac{1}{\bar{\chi}_{2} \chi_{1}+\theta^{2}}
\end{array}
$$

with $\chi_{1}=\chi_{1 x}+i \chi_{1 y}, \bar{\chi}_{2}=\chi_{2 x}-i \chi_{2 y}$. those integrals can be readily taken, as well:

$$
\begin{aligned}
& r\left(\chi_{1}, \chi_{2}, 0, \omega T\right) \\
& =-\mathrm{Ci}\left(\frac{\omega T \chi_{1}^{2}}{2}\right)-\mathrm{Ci}\left(\frac{\omega T \chi_{2}^{2}}{2}\right) \\
& +\mathfrak{R e}\left\{\operatorname { c o s } \frac { \omega T \chi _ { 1 } \overline { \chi } _ { 2 } } { 2 } \left(\mathrm{Ci}\left[\frac{\omega T}{2}\left(\chi_{1}^{2}+\chi_{1} \bar{\chi}_{2}\right)\right]\right.\right. \\
& \left.+\mathrm{Ci}\left[\frac{\omega T}{2}\left(\chi_{2}^{2}+\chi_{1} \bar{\chi}_{2}\right)\right]-\mathrm{Ci}\left[\frac{\omega T}{2} \chi_{1} \bar{\chi}_{2}\right]\right) \\
& +\sin \frac{\omega T \chi_{1} \bar{\chi}_{2}}{2}\left(\operatorname{si}\left[\frac{\omega T}{2}\left(\chi_{1}^{2}+\chi_{1} \bar{\chi}_{2}\right)\right]\right. \\
& \left.\left.+\operatorname{si}\left[\frac{\omega T}{2}\left(\chi_{2}^{2}+\chi_{1} \bar{\chi}_{2}\right)\right]-\operatorname{si}\left[\frac{\omega T}{2} \chi_{1} \bar{\chi}_{2}\right]\right)\right\} .
\end{aligned}
$$

This function must be added to $6 \mathrm{~b}$, with a proviso that owing to the admitted neglect of $\gamma^{-1}$, the validity of approximation $13 \mathrm{~b}$ is restricted to the domain $\omega T \lesssim \chi^{-2}$.

At $\gamma^{-2} \ll \omega T \ll \gamma \chi^{-1}$, approximation 13 devolves to decreasing harmonic oscillations:

$$
\begin{aligned}
r\left(\chi_{1}, \chi_{2}, 0, \omega T\right) & \simeq \frac{2}{\omega T}\left(\mathfrak{R e} \frac{1}{\chi_{1}^{2}+\chi_{1} \bar{\chi}_{2}}-\frac{1}{\chi_{1}^{2}}\right) \sin \frac{\omega T \chi_{1}^{2}}{2} \\
& +\frac{2}{\omega T}\left(\mathfrak{R e} \frac{1}{\chi_{2}^{2}+\chi_{1} \bar{\chi}_{2}}-\frac{1}{\chi_{2}^{2}}\right) \sin \frac{\omega T \chi_{2}^{2}}{2}
\end{aligned}
$$

But their decrease rate appears to be too slow, so in the far asymptotics, this approximation needs to be corrected. To this end, instead of considering the full integrand in (7), it suffices to single out only its most singular parts - vicinities of points $\boldsymbol{\theta}=-\chi_{1}$ and $\boldsymbol{\theta}=\chi_{2}$. The calculation then gives

$$
\begin{aligned}
& r\left(\chi_{1}, \chi_{2}, \gamma^{-1}, \omega T\right) \simeq \frac{1}{\pi}\left(\frac{\chi_{1}+\chi_{2}}{\left(\chi_{1}+\chi_{2}\right)^{2}}-\frac{\chi_{1}}{\chi_{1}^{2}}\right) \\
& \cdot \int d^{2} \theta \frac{\boldsymbol{\theta}+\chi_{1}}{\gamma^{-2}+\left(\boldsymbol{\theta}+\chi_{1}\right)^{2}} \cos \frac{\omega T}{2}\left[\chi_{1}^{2}-2 \chi_{1} \cdot\left(\boldsymbol{\theta}+\chi_{1}\right)\right] \\
& +\frac{1}{\pi}\left(\frac{\chi_{2}}{\chi_{2}^{2}}-\frac{\chi_{1}+\chi_{2}}{\left(\chi_{1}+\chi_{2}\right)^{2}}\right) \\
& \cdot \int d^{2} \theta \frac{\boldsymbol{\theta}-\chi_{2}}{\gamma^{-2}+\left(\boldsymbol{\theta}-\chi_{2}\right)^{2}} \cos \frac{\omega T}{2}\left[\chi_{2}^{2}+2 \chi_{2} \cdot\left(\boldsymbol{\theta}-\chi_{2}\right)\right] \\
& \simeq-\frac{\chi_{2} \cdot\left(\chi_{1}+\chi_{2}\right)}{\left(\chi_{1}+\chi_{2}\right)^{2}} \frac{2}{\gamma \chi_{1}} \sin \frac{\omega T \chi_{1}^{2}}{2} K_{1}\left(\frac{\omega T \chi_{1}}{\gamma}\right) \\
& -\frac{\chi_{1} \cdot\left(\chi_{1}+\chi_{2}\right)}{\left(\chi_{1}+\chi_{2}\right)^{2}} \frac{2}{\gamma \chi_{2}} \sin \frac{\omega T \chi_{2}^{2}}{2} K_{1}\left(\frac{\omega T \chi_{2}}{\gamma}\right)
\end{aligned}
$$

with $K_{1}$ the Macdonald function [20]. At relatively low $\omega\left(\chi^{-2} \ll \omega T \ll \gamma \chi^{-1}\right)$, when $K_{1}\left(\frac{\omega T \chi}{\gamma}\right) \rightarrow \frac{\gamma}{\omega T \chi}$, form (15) reduces to the high- $\omega$ asymptotics 14 of Eq. $13 \mathrm{~b}$. 
Thus, equations $13 \mathrm{~b}$ and 15 can be unified by writing

$$
\begin{aligned}
& r\left(\chi_{1}, \chi_{2}, \gamma^{-1}, \omega T\right) \\
& \simeq A_{1}\left(\frac{\omega T \chi_{1}^{2}}{2}, \frac{\omega T \chi_{1} \chi_{2}}{2} e^{i \varphi_{12}}\right) F_{\perp}\left(\frac{\omega T \chi_{1}}{\gamma}\right) \\
& +A_{1}\left(\frac{\omega T \chi_{2}^{2}}{2}, \frac{\omega T \chi_{1} \chi_{2}}{2} e^{i \varphi_{12}}\right) F_{\perp}\left(\frac{\omega T \chi_{2}}{\gamma}\right) \\
& +A_{2}\left(\frac{\omega T \chi_{1} \chi_{2}}{2} e^{i \varphi_{12}}\right) .
\end{aligned}
$$

Here

$$
\begin{gathered}
A_{1}\left(z_{1}, z_{2}\right)=-\operatorname{Ci}\left(z_{1}\right) \\
+\mathfrak{R e}\left\{\cos z_{2} \mathrm{Ci}\left(z_{1}+z_{2}\right)+\sin z_{2} \operatorname{si}\left(z_{1}+z_{2}\right)\right\}, \\
A_{2}(z)=-\mathfrak{R e}\{\cos z \operatorname{Ci}(z)+\sin z \operatorname{si}(z)\},
\end{gathered}
$$

may be interpreted as quasiantenna form factors, and

$$
F_{\perp}(z)=z K_{1}(z)
$$

being normalized by condition $F_{\perp}(0)=1$, as the electron's proper field form factor. In the next section, we will investigate its origin in more detail. Term $A_{2}$ in 16 . (stemming from the low- $\theta$ part of interference between the external lines) at $\omega T \chi^{2} \gg 1$ decreases faster than $A_{1}$ :

$$
A_{2}(z) \underset{z \rightarrow \infty}{\simeq} \mathfrak{R e} \frac{1}{z^{2}} .
$$

This coincides with the transient asymptotics of the original integral as a whole, so it appears unnecessary to endow $A_{2}$ with a suppressing form factor. ${ }^{8}$

Sine factors in 15 produce oscillations similar to those in (9), but are related with a different (electron scattering angle dependent) definition of the photon formation length:

$$
\sin \frac{\omega T \chi^{2}}{2}=\sin \frac{T}{l_{\chi}(\omega)}, \quad l_{\chi}(\omega) \underset{\gamma \chi \gg 1}{\simeq} \frac{2}{\omega \chi^{2}} .
$$

The reason why, in contrast to Eq. (9), we encounter here a sine instead of cosine dependence is that in Eq. 13a, cosine functions are integrated over photon emission angles from $\chi_{1}^{2}, \chi_{2}^{2}$ to infinity. Ultimately, those oscillations are damped by the exponentially decreasing factor $F_{\perp}$, but the damping proceeds slowly, since $F_{\perp}$ depends on $\omega$ on a scale which is $\gamma \chi$ times harder than the arguments of $A$ 's. So, there is enough room for the spectrum to make a number of visible oscillations.

\footnotetext{
8 With the account of $\mathcal{O}\left(\gamma^{-1}\right)$ corrections, 20 is actually followed by slow oscillations $-\frac{1}{\omega T \chi^{2}} \sin \frac{\omega T}{2 \gamma^{2}}$, which ensure that $\int_{0}^{\infty} d \omega A_{2}$ exactly equals zero, as is $\int_{0}^{\infty} d \omega A_{1}$. However, that faint contribution is virtually invisible against $I_{\mathrm{BH}}$ and $g$, so it seems harmless to neglect it entirely.
}

$\frac{\pi}{2 e^{2}} \frac{d I}{d \omega}$


FIG. 4: The spectrum of electromagnetic radiation from a doubly scattered electron. (a) Full spectrum for a case of scattering through equal angles $\chi_{1}=\chi_{2}=\chi, \chi=30 \gamma^{-1}$ (solid curve). Approximation 22 is virtually indistinguishable from this. The dot-dashed curve shows the behavior of asymptotics 23). Two separate regions of oscillatory behavior at intermediate and at high $\omega$ are determined by different photon formation lengths. (b) Soft region of the radiation spectrum, for a more general case $\left|\chi_{1}\right|=\left|\chi_{2}\right|=30 \gamma^{-1}$ and different values of azimuth $\varphi_{12}$. Solid curve, $\varphi_{12}=0$ (as in the upper figure). Dot-dashed, $\varphi_{12}=3 \pi / 4$ [evaluated by Eq. [22]. Red dashed curve, $\varphi_{12}=\pi$, corresponding to the jet overlap is evaluated by exact representations (6) and (7). In the latter case, the oscillations are anomalously large.

Contribution (16) may now be added to (8), and their sum

$$
\begin{aligned}
& \frac{d I}{d \omega} \underset{\chi_{1,2} \gg \gamma^{-1}}{\simeq} \frac{d I_{\mathrm{BH}}}{d \omega}\left(\gamma \chi_{1}\right)+\frac{d I_{\mathrm{BH}}}{d \omega}\left(\gamma \chi_{2}\right) \\
&+\frac{2 e^{2}}{\pi}\left[g\left(\frac{\omega T}{2 \gamma^{2}}\right)+\right. A_{1}\left(\frac{\omega T \chi_{1}^{2}}{2}, \frac{\omega T \chi_{1} \chi_{2}}{2} e^{i \varphi_{12}}\right) F_{\perp}\left(\frac{\omega T \chi_{1}}{\gamma}\right) \\
&+ A_{1}\left(\frac{\omega T \chi_{2}^{2}}{2}, \frac{\omega T \chi_{1} \chi_{2}}{2} e^{i \varphi_{12}}\right) F_{\perp}\left(\frac{\omega T \chi_{2}}{\gamma}\right) \\
&\left.+A_{2}\left(\frac{\omega T \chi_{1} \chi_{2}}{2} e^{i \varphi_{12}}\right)\right]
\end{aligned}
$$

gives a satisfactory approximation to the exact result 
for all $\omega$. Term $g$ here represents a hard contribution, whereas the residual quasiantenna terms represent the soft contribution, which is yet regulated by the appropriate proper field formfactors depending on $\omega$ on a scale intermediate between soft and hard. At $\omega \rightarrow 0$, logarithmic divergences of terms $g$ and $A_{2}$ mutually cancel, as they must, and up to terms linear in $\omega$, the result reads

$$
\frac{d I}{d \omega} \underset{\omega T \chi_{1,2}^{2} \ll 1}{\simeq} \frac{d I_{\mathrm{BH}}}{d \omega}\left(\gamma\left|\chi_{1}+\chi_{2}\right|\right)-e^{2} \omega T \frac{\chi_{1} \cdot \chi_{2}}{2} .
$$

Note that the last term here is negative when $\chi_{1} \cdot \chi_{2}>0$; hence, in the low- $\omega$ domain, the spectrum suppression can be nonmonotonous and dive below the infrared limiting value.

A typical resulting spectrum for significant electron deflection angles (which are let have equal values, $\chi_{1}=\chi_{2}$ ) is shown in Fig. 4(a). It displays oscillations in two nonoverlapping regions, with visibilities $\sim 1 / \ln \gamma^{2} \chi^{2}$. If $\left|\chi_{1}\right|$ and $\left|\chi_{2}\right|$ were unequal, according to Eq. (15), lowomega oscillations would involve two periods, becoming less regular. ${ }^{9}$ At lowest $\omega$, in Fig. 4 a there is a bump predicted by Eq. (23). In practice, a superficially similar bumplike structure often occurs due to transition radiation on solid target boundaries (see 10]). However, we do not include here any medium polarization effects, the bump being merely due to positive correlation between electron deflection angles.

Comparing solid and dot-dashed curves in Fig. 4(b), we can see that for the case of scattering angles coinciding by absolute value, low- $\omega$ oscillations are virtually independent of the azimuth between the scattering planes, given that in Eq. $15, \frac{\boldsymbol{\chi}_{1} \cdot\left(\boldsymbol{\chi}_{1}+\boldsymbol{\chi}_{2}\right)}{\left(\boldsymbol{\chi}_{1}+\boldsymbol{\chi}_{2}\right)^{2}}=\frac{\boldsymbol{\chi}_{2} \cdot\left(\boldsymbol{\chi}_{1}+\boldsymbol{\chi}_{2}\right)}{\left(\boldsymbol{\chi}_{1}+\boldsymbol{\chi}_{2}\right)^{2}}=\frac{1}{2}$. Anomalously large oscillations emerge, however, at $\chi_{2} \rightarrow-\chi_{1}$ (dashed curve). That corresponds to an overlap of radiation cones aligned with initial and final electron lines, and leads to breakdown of formula (15). Such a case is exceptional, and generally will be beyond the scope of the present paper.

The causal connection between directions of electron motion and that of photon emission means that together they form a jet. More precisely, in this process there are two categories of photons: intrajet (inside a jet) and interjet (between the jets), as is evident from Fig. 2. In quantum electrodynamics, in interpretations of radiative corrections integrated over $\omega$ and all components of $\boldsymbol{k}$, intrajet photons are generally called collinear, whereas low-frequency photons which do not have collinear properties (in our case - interjet, although they may incidentally propagate along one of the jet directions, as well) are called soft [19, 23. In their terms, internal line resonances in the hard spectral domain in Fig. 4(a) are due to "collinear-collinear" radiation interference (interference

\footnotetext{
9 If furthermore we average over an interval of $\chi_{1}$ and $\chi_{2}$ so large that $\delta \chi_{1,2} \gtrsim \chi_{1,2}$, soft oscillations would be washed out. That is why they were not discovered in works [1-4.
}

between photons generated by the electron in the intermediate state and emitted close to its velocity), whereas "radio" resonances in the soft domain are "soft-collinear" interference (when only one of the interfering photons is closely aligned with the initial or final electron line).

The notion of jets also helps elucidate why photon formation length (21) results from generic Eq. (1) by exactly substituting $\theta \rightarrow \chi$ : The emission angle for interfering photons is counted off from the direction of one of the electron lines (internal) to the direction of another (external) electron line, along which such photons are actually emitted, and the indeterminacy of the emission angle $\sim \gamma^{-1}$ is much smaller than its mean value $\chi$. This, though, does not completely specify the process geometry in position space yet. There also remains an issue why the proper field form factors, which are asymptotically exponential, depend on the absolute value of the deflection angle. Finally, our assumption that $l_{0}$ and $l_{\chi}$ are the photon formation lengths was actually not strictly proven within the approach of the present section. It thus deserves additional space-time considerations. In particular, one can anticipate the factorization property for the low- $\omega$ part also to be backed by some spatially causal reasons.

\section{IMPACT PARAMETER REPRESENTATION}

In this section, we will explore properties of transverse spatial variables for emitted photons. They must be Fourier-dual to the photon transverse wave vector, and actually be in the spirit of ray optics. If conditions of ray optics do apply, impact parameters ${ }^{10}$ should assume rather well-defined values characterizing preferable light rays.

Some complications emerge in this regard, however, since Eq. (2) involves not the local electromagnetic field, but the radiation emission amplitude. Besides that, the electromagnetic field is physically coupled to the electron, which arrives from and moves off to infinity. Nonetheless, well defined should be the notion of impact parameter of an electromagnetic wave with respect to the initial or to the final electron line. At that, since directions of electron motion along those lines differ, there will arise simultaneously two instead of one species of the impact parameter (in contrast to the Glauber description of short-wave scattering on finite obstacles, where a single definition for impact parameters is sufficient).

A formulation of the impact parameter view for the case of electron double scattering can be attained as follows. In representation (3), for each of the algebraic

10 Here we actually deal with photon emission rather than impact, but to stress the analogy with the equivalent photon method, we adopt the same terminology. 
terms in the brackets, apply transformation

$$
\frac{\boldsymbol{\theta}}{\gamma^{-2}+\theta^{2}}=\frac{i}{2 \pi} \int d^{2} \xi e^{i \boldsymbol{\theta} \cdot \boldsymbol{\xi}} \frac{\partial}{\partial \boldsymbol{\xi}} K_{0}\left(\frac{\xi}{\gamma}\right),
$$

[obtained from well known identity $\frac{1}{\gamma^{-2}+\theta^{2}}=$ $\frac{1}{2 \pi} \int d^{2} \xi e^{i \boldsymbol{\theta} \cdot \boldsymbol{\xi}} K_{0}\left(\frac{\xi}{\gamma}\right)$ by integrating by parts]. That leads to representation 4], which for our present purposes more conveniently casts as

$$
\begin{aligned}
\frac{d I}{d \omega} & =\frac{d I_{\mathrm{BH}}}{d \omega}\left(\gamma \chi_{1}\right)+\frac{d I_{\mathrm{BH}}}{d \omega}\left(\gamma \chi_{2}\right) \\
& -\frac{e^{2}}{\pi^{3} \omega T} \iint d^{2} \xi_{1} d^{2} \xi_{2} \frac{\partial}{\partial \boldsymbol{\xi}_{1}} K_{0}\left(\frac{\xi_{1}}{\gamma}\right) \cdot \frac{\partial}{\partial \boldsymbol{\xi}_{2}} K_{0}\left(\frac{\xi_{2}}{\gamma}\right) \\
& \times \mathfrak{I m}\left(1-e^{-i \boldsymbol{\chi}_{1} \cdot \boldsymbol{\xi}_{1}}\right)\left(1-e^{-i \boldsymbol{\chi}_{2} \cdot \boldsymbol{\xi}_{2}}\right) e^{-i \frac{\omega T}{2 \gamma^{2}}+i \frac{\left(\boldsymbol{\xi}_{1}-\boldsymbol{\xi}_{2}\right)^{2}}{2 \omega T}}
\end{aligned}
$$

The impact parameter here is represented by $\boldsymbol{\xi} / \omega$ rather than $\boldsymbol{\xi}$ alone (the latter is dimensionless). Specifically, $\boldsymbol{\xi}_{1} / \omega$ is the impact parameter with respect to the first scattering vertex, and $\boldsymbol{\xi}_{2} / \omega$ is that with respect to the second vertex. We will see shortly that this approach is largely similar to the equivalent photon one [14, with a proviso that from the outset we deal with strictly real photons, and do not restrict ourselves to dipole approximation.

Examining structure 24, it is evident that at $\chi \gg$ $\gamma^{-1}$, terms $e^{-i \boldsymbol{\chi}_{1} \cdot \boldsymbol{\xi}_{1}}$ and $e^{-i \boldsymbol{\chi}_{2} \cdot \boldsymbol{\xi}_{2}}$ are rapidly oscillating. Thus, the main contribution to the integral is brought by the $\chi$-independent term

$$
\begin{gathered}
g\left(\frac{\omega T}{2 \gamma^{2}}\right)=\frac{1}{2 \pi^{2} \omega T} \iint d^{2} \xi_{1} d^{2} \xi_{2} \frac{\partial}{\partial \boldsymbol{\xi}_{1}} K_{0}\left(\frac{\xi_{1}}{\gamma}\right) \cdot \frac{\partial}{\partial \boldsymbol{\xi}_{2}} K_{0}\left(\frac{\xi_{2}}{\gamma}\right) \\
\times \sin \left[\frac{\omega T}{2 \gamma^{2}}-\frac{\left(\boldsymbol{\xi}_{1}-\boldsymbol{\xi}_{2}\right)^{2}}{2 \omega T}\right],
\end{gathered}
$$

which can be shown (e.g., by returning to the emission angle representation) to coincide with (6). The high- $\omega$ asymptotics of Eq. 25 can be derived by noting that therein typical contributing $\xi_{1}, \xi_{2}$ are small. It is, however, impossible to entirely neglect term $\frac{\left(\boldsymbol{\xi}_{1}-\boldsymbol{\xi}_{2}\right)^{2}}{2 \omega T}$ in the argument of the sine, because then the integrals over $\boldsymbol{\xi}_{1}$ and $\boldsymbol{\xi}_{2}$ would vanish. Expanding through the next-toleading order

$$
\begin{array}{r}
\sin \left[\frac{\omega T}{2 \gamma^{2}}-\frac{\left(\boldsymbol{\xi}_{1}-\boldsymbol{\xi}_{2}\right)^{2}}{2 \omega T}\right] \simeq \sin \left(\frac{\omega T}{2 \gamma^{2}}-\frac{\boldsymbol{\xi}_{1}^{2}+\boldsymbol{\xi}_{2}^{2}}{2 \omega T}\right) \\
+\frac{\boldsymbol{\xi}_{1} \cdot \boldsymbol{\xi}_{2}}{\omega T} \cos \frac{\omega T}{2 \gamma^{2}}
\end{array}
$$

and inserting this to 25 reproduces Eq. (9).

In contrast, at low $\omega$, we know from Sec. III that the rest of the interference terms become important, as well, but here it is justified to set $\gamma^{-1} \rightarrow 0$. From the standpoint of representation 24, that owes to the smallness of contributing $\xi_{1}, \xi_{2}$. Integration in

$$
\begin{aligned}
& r\left(\boldsymbol{\chi}_{1}, \boldsymbol{\chi}_{2}, 0, \omega T\right)=\frac{1}{2 \pi^{2} \omega T} \iint d^{2} \xi_{1} d^{2} \xi_{2} \frac{\boldsymbol{\xi}_{1}}{\xi_{1}^{2}} \cdot \frac{\boldsymbol{\xi}_{2}}{\xi_{2}^{2}} \\
& \quad \times \mathfrak{I m}\left(e^{-i \boldsymbol{\chi}_{1} \cdot \boldsymbol{\xi}_{1}}+e^{-i \boldsymbol{\chi}_{2} \cdot \boldsymbol{\xi}_{2}}-e^{-i \boldsymbol{\chi}_{1} \cdot \boldsymbol{\xi}_{1}-i \boldsymbol{\chi}_{2} \cdot \boldsymbol{\xi}_{2}}\right) e^{i \frac{\left(\boldsymbol{\xi}_{1}-\boldsymbol{\xi}_{2}\right)^{2}}{2 \omega T}}
\end{aligned}
$$

with the use of same conformal properties (11) and 12 gives back the same integral sine and cosine representation 13b.

Our main interest, however, lies in the case $\omega T \chi^{2} \gg 1$, since impact parameters then assume rather sharp values, and suggest a direct physical interpretation. We thus examine integral (24) under condition $\chi^{-2} \ll \omega T \ll \chi^{-1} \gamma$ more closely.

To begin with, in one of the $\chi$-dependent terms of (24),

$$
\begin{aligned}
\frac{1}{2 \pi^{2} \omega T} \mathfrak{I m} \iint d^{2} \xi_{1} d^{2} \xi_{2} \frac{\partial}{\partial \boldsymbol{\xi}_{1}} K_{0} & \left(\frac{\xi_{1}}{\gamma}\right) \cdot \frac{\partial}{\partial \boldsymbol{\xi}_{2}} K_{0}\left(\frac{\xi_{2}}{\gamma}\right) \\
& \times e^{-i \boldsymbol{\chi}_{1} \cdot \boldsymbol{\xi}_{1}+\frac{i}{2 \omega T}\left(\boldsymbol{\xi}_{1}-\boldsymbol{\xi}_{2}\right)^{2}}
\end{aligned}
$$

the dominant contribution stems from small $\xi_{2}$, allowing it to be approximated as

$$
\begin{gathered}
-\frac{1}{2 \pi^{2} \omega T} \mathfrak{I m} \int d^{2} \xi_{1} \frac{\partial}{\partial \boldsymbol{\xi}_{1}} K_{0}\left(\frac{\xi_{1}}{\gamma}\right) e^{-i \boldsymbol{\chi}_{1} \cdot \boldsymbol{\xi}_{1}+\frac{i}{2 \omega T} \xi_{1}^{2}} \\
\cdot \int d^{2} \xi_{2} \frac{\boldsymbol{\xi}_{2}}{\xi_{2}^{2}} e^{-\frac{i}{\omega T} \boldsymbol{\xi}_{1} \cdot \boldsymbol{\xi}_{2}}
\end{gathered}
$$

Here the integral over $\boldsymbol{\xi}_{2}$ equals $\int d^{2} \xi_{2} \frac{\boldsymbol{\xi}_{2}}{\xi_{2}^{2}} e^{-\frac{i}{\omega T} \boldsymbol{\xi}_{1} \cdot \boldsymbol{\xi}_{2}}=$ $\frac{2 \pi \omega T}{i} \frac{\xi_{1}}{\xi_{1}^{2}}$, and that over $\boldsymbol{\xi}_{1}$ engages a rapidly oscillating exponential $e^{-i \boldsymbol{\chi}_{1} \cdot \boldsymbol{\xi}_{1}+\frac{i}{2 \omega T} \xi_{1}^{2}}=e^{\frac{i}{2 \omega T}\left(\boldsymbol{\xi}_{1}-\omega T \boldsymbol{\chi}_{1}\right)^{2}-\frac{i}{2} \omega T \chi_{1}^{2}}$, which has a stationary phase point at $\boldsymbol{\xi}_{1}=\omega T \chi_{1}$. That effectively fixes $\boldsymbol{\xi}_{1}$ in other factors at this value:

$$
\frac{\partial}{\partial \boldsymbol{\xi}_{1}} K_{0}\left(\frac{\xi_{1}}{\gamma}\right) \cdot \frac{\boldsymbol{\xi}_{1}}{\xi_{1}^{2}} \rightarrow-\frac{1}{\gamma \omega T \chi_{1}} K_{1}\left(\frac{\omega T \chi_{1}}{\gamma}\right)
$$

The result of integration in 27) then equals

$$
-\frac{2}{\gamma \chi_{1}} \sin \frac{\omega T \chi_{1}^{2}}{2} K_{1}\left(\frac{\omega T \chi_{1}}{\gamma}\right)
$$

Similarly, the integral containing $e^{-i \boldsymbol{\chi}_{2} \cdot \boldsymbol{\xi}_{2}}$ reduces to

$$
\begin{aligned}
& \frac{1}{2 \pi^{2} \omega T} \mathfrak{I m} \iint d^{2} \xi_{1} d^{2} \xi_{2} \frac{\partial}{\partial \boldsymbol{\xi}_{1}} K_{0}\left(\frac{\xi_{1}}{\gamma}\right) \cdot \frac{\partial}{\partial \boldsymbol{\xi}_{2}} K_{0}\left(\frac{\xi_{2}}{\gamma}\right) \\
& \simeq \quad \times e^{-i \boldsymbol{\chi}_{2} \cdot \boldsymbol{\xi}_{2}+\frac{i}{2 \omega T}\left(\boldsymbol{\xi}_{1}-\boldsymbol{\xi}_{2}\right)^{2}} \\
& \simeq-\frac{2}{\gamma \chi_{2}} \sin \frac{\omega T \chi_{2}^{2}}{2} K_{1}\left(\frac{\omega T \chi_{2}}{\gamma}\right)
\end{aligned}
$$

Finally, the integral containing $e^{-i \boldsymbol{\chi}_{1} \cdot \boldsymbol{\xi}_{1}-i \boldsymbol{\chi}_{2} \cdot \boldsymbol{\xi}_{2}}$ receives two dominant contributions, in one of which $\boldsymbol{\xi}_{1}$ is small while $\boldsymbol{\xi}_{2}$ is finite, and in the other one $\boldsymbol{\xi}_{2}$ is small while 
$\boldsymbol{\xi}_{1}$ is finite:

$$
\begin{array}{r}
-\frac{1}{2 \pi^{2} \omega T} \mathfrak{I m} \iint d^{2} \xi_{1} d^{2} \xi_{2} \frac{\partial}{\partial \boldsymbol{\xi}_{1}} K_{0}\left(\frac{\xi_{1}}{\gamma}\right) \cdot \frac{\partial}{\partial \boldsymbol{\xi}_{2}} K_{0}\left(\frac{\xi_{2}}{\gamma}\right) \\
\times e^{-i \boldsymbol{\chi}_{1} \cdot \boldsymbol{\xi}_{1}-i \boldsymbol{\chi}_{2} \cdot \boldsymbol{\xi}_{2}+\frac{i}{2 \omega T}\left(\boldsymbol{\xi}_{1}-\boldsymbol{\xi}_{2}\right)^{2}} \\
\simeq \frac{1}{2 \pi^{2} \omega T} \mathfrak{I m} \int d^{2} \xi_{2} e^{-i \boldsymbol{\chi}_{2} \cdot \boldsymbol{\xi}_{2}+\frac{i}{2 \omega T} \xi_{2}^{2}} \frac{\partial}{\partial \boldsymbol{\xi}_{2}} K_{0}\left(\frac{\xi_{2}}{\gamma}\right) \\
\cdot \int d^{2} \xi_{1} \frac{\boldsymbol{\xi}_{1}}{\xi_{1}^{2}} e^{-i\left(\boldsymbol{\chi}_{1}+\frac{\xi_{2}}{\omega T}\right) \cdot \boldsymbol{\xi}_{1}} \\
+\frac{1}{2 \pi^{2} \omega T} \mathfrak{I m} \int d^{2} \xi_{1} e^{-i \boldsymbol{\chi}_{1} \cdot \boldsymbol{\xi}_{1}+\frac{i}{2 \omega T} \xi_{1}^{2}} \frac{\partial}{\partial \boldsymbol{\xi}_{1}} K_{0}\left(\frac{\xi_{1}}{\gamma}\right) \\
\cdot \int d^{2} \xi_{2} \frac{\boldsymbol{\xi}_{2}}{\xi_{2}^{2}} e^{-i\left(\boldsymbol{\chi}_{2}+\frac{\boldsymbol{\xi}_{1}}{\omega T}\right) \cdot \boldsymbol{\xi}_{2}} .
\end{array}
$$

Those integrals can be evaluated in exactly the same way as above, giving

$$
\begin{array}{r}
-\frac{1}{2 \pi^{2} \omega T} \\
\mathfrak{I m} \iint d^{2} \xi_{1} d^{2} \xi_{2} \frac{\partial}{\partial \boldsymbol{\xi}_{1}} K_{0}\left(\frac{\xi_{1}}{\gamma}\right) \cdot \frac{\partial}{\partial \boldsymbol{\xi}_{2}} K_{0}\left(\frac{\xi_{2}}{\gamma}\right) \\
\quad \times e^{-i \boldsymbol{\chi}_{1} \cdot \boldsymbol{\xi}_{1}-i \boldsymbol{\chi}_{2} \cdot \boldsymbol{\xi}_{2}+\frac{i}{2 \omega T}\left(\boldsymbol{\xi}_{1}-\boldsymbol{\xi}_{2}\right)^{2}} \\
\simeq \frac{2}{\gamma \chi_{2}} \frac{\boldsymbol{\chi}_{2} \cdot\left(\boldsymbol{\chi}_{1}+\boldsymbol{\chi}_{2}\right)}{\left(\boldsymbol{\chi}_{1}+\boldsymbol{\chi}_{2}\right)^{2}} \sin \frac{\omega T \chi_{2}^{2}}{2} K_{1}\left(\frac{\omega T \chi_{2}}{\gamma}\right) \\
+\frac{2}{\gamma \chi_{1}} \frac{\boldsymbol{\chi}_{1} \cdot\left(\boldsymbol{\chi}_{1}+\boldsymbol{\chi}_{2}\right)}{\left(\chi_{1}+\chi_{2}\right)^{2}} \sin \frac{\omega T \chi_{1}^{2}}{2} K_{1}\left(\frac{\omega T \chi_{1}}{\gamma}\right) .
\end{array}
$$

Combined with (28) and (29), it leads to result (15).

The derivation offered above elucidates the geometrical origin of proper field form factor $F_{\perp}$ : It corresponds to the impact parameter distribution amplitude for one of the intra jet photons, at a fixed impact parameter $\Delta \boldsymbol{b}=\boldsymbol{\xi}_{1} / \omega=T \chi_{1}$ determined by the difference of impact parameters between the vertices, since emission of an interjet photon is completed in a relatively small spatial domain. The corresponding form factors in Eq. 222,

$$
F_{\perp}\left(\frac{\omega T \chi}{\gamma}\right)=F_{\perp}\left(\frac{\Delta b(T, \chi)}{l_{\perp}(\gamma, \omega)}\right)
$$

depend on the ratio of the aforementioned $\Delta b$ and

$$
l_{\perp}(\omega)=\frac{\gamma}{\omega}
$$

which thus plays the role of a "transverse coherence length". One actually recognizes in 30 nothing but the typical transverse scale for the electric field of an ultrarelativistic particle, well known within, e.g., the equivalent photon approach [14. In capacity of a coherence length in our problem, it does not give rise to a new type of oscillations, but describes damping of an old one.

The analogy with the equivalent photon approach is strengthened by observing that Fourier expansion of the transverse component of the electric or magnetic field of ultrarelativistic electron $E_{\perp}(b, z, t)=\frac{Z e \gamma b}{\left[b^{2}+\gamma^{2}(z-v t)^{2}\right]^{3 / 2}}$ gives

$$
\int_{-\infty}^{\infty} d t e^{i \omega t} E_{\perp}(b, 0, t)=\frac{2 Z e}{v b} F_{\perp}\left(\frac{\omega b}{v \gamma}\right)
$$
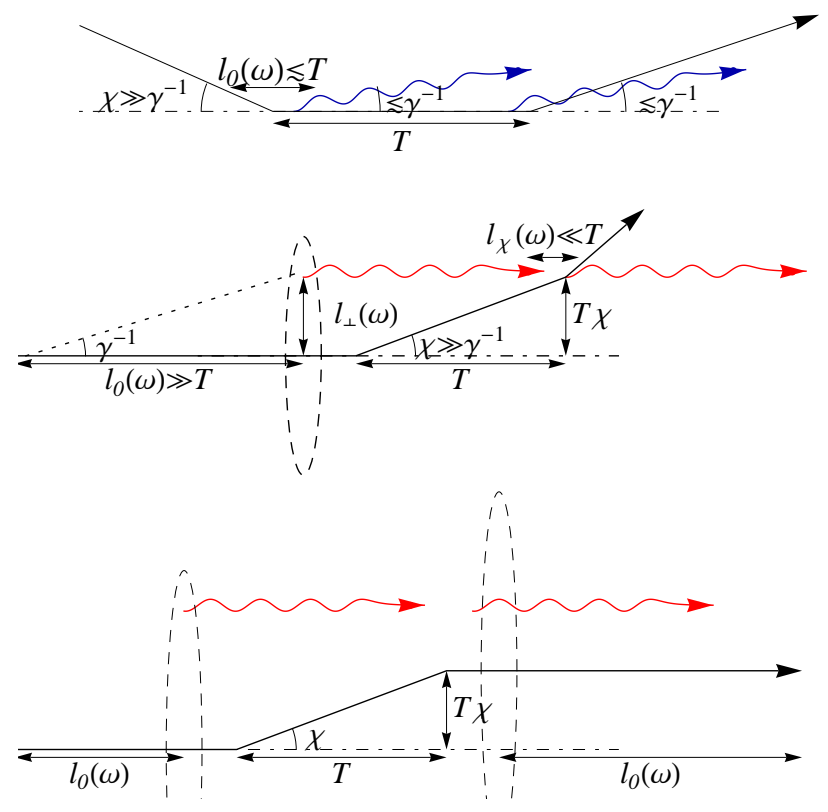

(c)

FIG. 5: (a) High- $\omega$ interference diagram. (b) Low- $\omega$ interference diagram, in the absence of jet overlap. In this case, there is also a cross diagram, in which noncollinear photons are emitted from the first scattering vertex, and collinear ones from the final electron line. The condition of interference between collinear and noncollinear photons, besides the coincidence of emission directions, is the equality of impact parameters: $l_{\perp}(\omega)=T \chi$. (c) Low- $\omega$ interference diagram in the case of jet overlap $\left(\chi_{2}=-\chi_{1}\right)$. For interfering collinear photons in this case, only the difference of impact parameters is fixed.

where $v \rightarrow 1$ in the ultrarelativistic case, and form factor $F_{\perp}$, absorbing all the $\omega$-dependence, coinciding with $(19)$.

It may be instructive to compare virtues of the formalism of the present section with that in Sec. III] In the hard spectral domain, the interference of radiation is described well enough by the photon emission angle representation of Sec. III] demonstrating that photons participating in the interference emerge under fairly well-defined angles close to the intermediate electron direction of motion [see Fig. 5(a)]. The range of contributing angles shrinks reciprocally with the increase of $\omega$ :

$$
(\delta \theta)^{2} \sim \frac{2}{\omega T},
$$

producing the power-law falloff factor in the spectral oscillations. In the soft domain, however, a clearer physical picture is offered by the impact parameter representation, revealing that in addition to emission of the interfering photons parallel to the initial or final electron line [as is already clear in the emission angle representation of Sec. III particularly Fig. 2(b)], in the configuration space they must yet nearly belong to a ray going parallel to the external electron line at a distance such that it passes through the opposite vertex (see Fig. 5b). At that, the fraction of such photons, quantified by the spread of the contributing impact parameters in integral (24), 
$\delta(\xi / \omega)^{2} \sim T / \omega$, appears to be significant compared to $(T \chi)^{2}$ if $\omega T \sim \chi^{-2}$. With the increase of $\omega$, this fraction decreases, because of the exponential decrease of the intrajet photon wave function at large impact parameters.

The impact parameter view also gives better understanding of the condition of applicability of classical electrodynamics for the present process: The requirement of negligibility of photon recoil, $\omega \ll E / \hbar$, in combination with our estimate $\omega \sim \frac{1}{T \chi^{2}}$ for typical photon frequencies in the soft interference domain expresses as

$$
p_{\perp} \Delta b \gg \hbar,
$$

where $p_{\perp}=E \chi$ is the electron transverse momentum, while $\Delta b=T \chi$, as before. Hence, the semiclassical tractability of soft photon emission in the given process is equivalent to the semiclassicality of transverse motion of the electron within the intermediate trajectory segment.

\section{TIME EVOLUTION}

To corroborate our conjecture that $l_{0}$ and $l_{\chi}$ are the true formation lengths for interfering photons in the corresponding spectral domains, it is necessary to provide also some longitudinal coordinate considerations. It is difficult to simultaneously handle all three spatial coordinates and the photon emission frequency, so we will restrict ourselves in this section to a simplified treatment only in terms of the photon emission times, which are manifestly present in formula 2 .

An emission time representation for the radiation spectrum derives from (2) by performing prior integration over the radiation angles, which is manageable for a generic electron trajectory $\boldsymbol{r}(t)$. That relinquishes the issue of the photon formation length dependence on the emission angles, but instead introduces its direct dependence on the electron deflection angles. Moreover, since we now encounter a double time integral, coherence lengths duplicate, and there may also occur a correlation between the emission times. We will expound the corresponding procedure as briefly as possible.

In the photon emission spectrum, two-time correlation on the electron trajectory, in effect, is mediated by the photon propagator (see the Appendix), which depends on the electromagnetic field gauge. The simplest for use is Feynman gauge, in which the angle-integral radiation spectrum reads:

$$
\begin{aligned}
\frac{d I}{d \omega}=\omega \frac{e^{2}}{\pi} \int_{0}^{\infty} \frac{d \tau}{\tau} \int_{-\infty}^{\infty} d t_{2} & \left(\left\{\gamma^{-2}+\frac{1}{2}\left[\boldsymbol{v}\left(t_{2}\right)-\boldsymbol{v}\left(t_{2}-\tau\right)\right]^{2}\right\}\right. \\
\times & \sin \omega\left[\tau-\left|\boldsymbol{r}\left(t_{2}\right)-\boldsymbol{r}\left(t_{2}-\tau\right)\right|\right] \\
& \left.-\gamma^{-2} \sin \omega(1-v) \tau\right),
\end{aligned}
$$

order to make the integrand vanish for a uniform and rectilinear charge motion. For $|\boldsymbol{v}(t)|=$ const, this term is independent of the particle trajectory detail.

Representation (32) is commonly used in practice, so we will embark on it, too. Although it involves a different gauge compared to preceding sections, key elements, contained in the phase of the sine, are gauge independent.

Given the presence of two integration times, for analysis of the coherence, the integral should be reduced to a single one with an oscillatory integrand, leading contributions from which may formally be related with coherence properties. In capacity of such a variable in the present case one can take a time ratio, which, just like photon emission angles, is related with the process geometry. That is tantamount to dispensing with kinematic definition (1), and dealing with a more dynamical one.

For simplicity, in this section we will confine ourselves to calculations for symmetric and coplanar electron scattering, when

$$
\chi_{1}=\chi_{2}
$$

That will suffice for demonstration of relevance of coherence length notions, and will also expose similarities with other problems in which the electron motion is planar, e.g., synchrotron radiation in a finite magnet.

Since in our case the electron trajectory is rectilinear in each of the three intervals separated by the two scattering points, in integral (32) nonzero are only mutual interference terms between those intervals. Moreover, owing to the symmetry of the trajectory with respect to its middle point, interference integral of the inner part with the initial part is the same as that with the final part, $\mathcal{I}_{i e}=\mathcal{I}_{e i}$ (see Fig. 6). Thus, the spectrum is comprised merely by two essentially different contributions:

$$
\frac{d I}{d \omega}=\frac{2 e^{2}}{\pi}\left(\mathcal{I}_{e e}+2 \mathcal{I}_{e i}\right)
$$

In the ultrarelativistic approximation,

$$
\begin{aligned}
& \mathcal{I}_{e e}=\frac{\omega}{2 \gamma^{2}} \int_{T / 2}^{\infty} d t_{2} \int_{t_{2}+T / 2}^{\infty} \frac{d \tau}{\tau}\left\{\left(1+2 \gamma^{2} \chi^{2}\right)\right. \\
& \left.\times \sin \omega\left[\frac{\tau}{2 \gamma^{2}}+\frac{\chi^{2}}{2}\left(4 t_{2}\left(1-\frac{t_{2}}{\tau}\right)-T\right)\right]-\sin \frac{\omega \tau}{2 \gamma^{2}}\right\}(34)
\end{aligned}
$$

with $t_{2}$ being counted off from the midpoint of the intermediate time interval, and

$$
\begin{aligned}
\mathcal{I}_{e i}= & \frac{\omega}{2 \gamma^{2}} \int_{0}^{T} d t_{2}^{\prime} \int_{t_{2}^{\prime}}^{\infty} \frac{d \tau}{\tau}\left\{\left(1+\gamma^{2} \chi^{2} / 2\right)\right. \\
& \left.\times \sin \omega\left[\frac{\tau}{2 \gamma^{2}}+\frac{\chi^{2}}{2} t_{2}^{\prime}\left(1-\frac{t_{2}^{\prime}}{\tau}\right)\right]-\sin \frac{\omega \tau}{2 \gamma^{2}}\right\}
\end{aligned}
$$

with $t_{2}^{\prime}=t_{2}+T / 2$ counted off from the point of first scattering. Terms non-linear in times in the arguments

with $\tau=t_{2}-t_{1}$. The coefficient in the argument of the
last sine here is chosen based on convenience reasons, in 


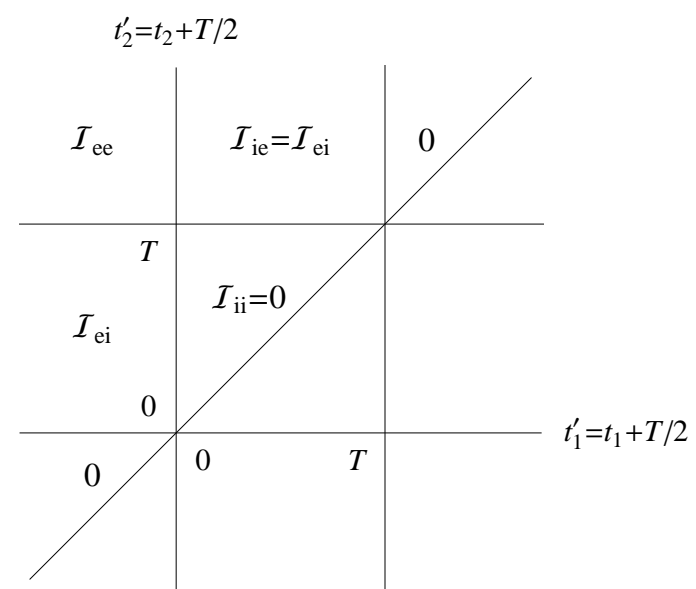

FIG. 6: Domains of continuity of the integrand of Eq. 32 in the double time plane [areas of definition of integrands of partial integrals (34) and (35)].

of sine functions originate from transverse coordinate difference, as we expand

$$
\begin{gathered}
v \tau-\left|\boldsymbol{r}\left(t_{2}\right)-\boldsymbol{r}\left(t_{2}-\tau\right)\right| \simeq \frac{1}{2 v \tau}\left\{v^{2} \tau^{2}-\left[\int_{t_{2}-\tau}^{t_{2}} d t \boldsymbol{v}(t)\right]^{2}\right\} \\
\simeq \frac{1}{2 v}\left\{\int_{t_{2}-\tau}^{t_{2}} d t \boldsymbol{v}_{\perp}^{2}(t)-\frac{1}{\tau}\left[\int_{t_{2}-\tau}^{t_{2}} d t \boldsymbol{v}_{\perp}(t)\right]^{2}\right\} .
\end{gathered}
$$

Note that the latter expression in terms of transverse velocity components, through which the longitudinal component expresses, as well, is invariant under small rotations of the Cartesian frame.

The advantage of choosing the origin for variables $t_{2}$ and $t_{2}^{\prime}$ in Eqs. (34) and (35) in different points (points of crossing of the corresponding rectilinear segments) consists in rendering the nonlinear terms in the phase scale invariant. ${ }^{11}$ That enables exact integration over one of the time variables, by introducing ratio $w=2 t_{2} / \tau$ instead of $t_{2}$ for $\mathcal{I}_{e e}$, and $w=t_{2}^{\prime} / \tau$ instead of $t_{2}^{\prime}$ for $\mathcal{I}_{e i}$. Integrations over $\tau$ then reduce to that of a sine with a

11 If the electron scattering angles are not coplanar, one has to perform a complex shift of the variable $t_{2}$. Thereby, complex integrals over $w$ reduce to a form analogous to Eq. $13 \mathrm{~b}$. linear argument, yielding: ${ }^{12}$

$$
\begin{aligned}
\mathcal{I}_{e e}=\frac{\omega}{4 \gamma^{2}} & \int_{0}^{2} d w \int_{\max \left\{\frac{T}{w}, \frac{T}{2-w}\right\}}^{\infty} d \tau\left\{\left(1+2 \gamma^{2} \chi^{2}\right)\right. \\
& \left.\times \sin \omega\left[\frac{\tau}{2 \gamma^{2}}+\frac{\chi^{2}}{2}[\tau w(2-w)-T]\right]-\sin \frac{\omega \tau}{2 \gamma^{2}}\right\} \\
=\int_{0}^{1} d w\left\{\frac{1+2 \gamma^{2} \chi^{2}}{1+\gamma^{2} \chi^{2} w(2-w)} \cos \frac{\omega T}{2 \gamma^{2}}\left[\frac{1}{w}+\gamma^{2} \chi^{2}(1-w)\right]\right. & \left.-\cos \frac{\omega T}{2 \gamma^{2} w}\right\}
\end{aligned}
$$

and

$$
\begin{aligned}
& \mathcal{I}_{e i}=\frac{\omega}{2 \gamma^{2}} \int_{0}^{1} d w \int_{0}^{T / w} d \tau\left\{\left(1+\gamma^{2} \chi^{2} / 2\right)\right. \\
& \left.\quad \times \sin \frac{\omega \tau}{2 \gamma^{2}}\left[1+\gamma^{2} \chi^{2} w(1-w)\right]-\sin \frac{\omega \tau}{2 \gamma^{2}}\right\} \\
& =\int_{0}^{1} d w\left\{\cos \frac{\omega T}{2 \gamma^{2} w}-1\right. \\
& \left.+\frac{1+\gamma^{2} \chi^{2} / 2}{1+\gamma^{2} \chi^{2} w(1-w)}\left(1-\cos \frac{\omega T}{2 \gamma^{2}}\left[\frac{1}{w}+\gamma^{2} \chi^{2}(1-w)\right]\right)\right\} .
\end{aligned}
$$

Integrals $37 \mathrm{~b}$ and $38 \mathrm{~b}$ span the same integration interval, and involve identical cosine factors, so they may reasonably be combined. Then, there arise significant cancellations between the prefactors, which can be explicated by splitting algebraic factors into simple fractions:

$$
\begin{aligned}
\frac{d I}{d \omega}=2 \frac{d I_{\mathrm{BH}}}{d \omega}(\gamma \chi) & +\frac{2 e^{2}}{\pi} \int_{0}^{1} d w\left\{\cos \frac{\omega T}{2 \gamma^{2} w}\right. \\
+\left(\frac{1}{w+\frac{1}{2 \gamma^{2} \chi^{2}}}-\right. & \left.\frac{1}{w+\frac{1}{\gamma^{2} \chi^{2}}}+\frac{1}{2-w}-\frac{1}{1+\frac{1}{\gamma^{2} \chi^{2}}-w}\right) \\
& \left.\times \cos \frac{\omega T}{2 \gamma^{2}}\left[\frac{1}{w}+\gamma^{2} \chi^{2}(1-w)\right]\right\}
\end{aligned}
$$

Here the noninterference contribution ${ }^{13}$

$$
\frac{d I_{\mathrm{BH}}}{d \omega}(\gamma \chi)=\frac{2 e^{2}}{\pi}\left(\int_{0}^{1} d w \frac{1+\gamma^{2} \chi^{2} / 2}{1+\gamma^{2} \chi^{2} w(1-w)}-1\right)
$$

12 In $37 \mathrm{~b}$ we exploited the symmetry of the integrand, by virtue of which $\int_{1}^{2} d w \ldots=\int_{0}^{1} d w \ldots$

13 Structure 40 emerges also when evaluating integral 4a by Feynman parametrization. Relationships of Feynman parameters with time variables were formerly found in quantum field theory [21]. 
actually coincides with $4 \mathrm{a}$.

The rest is straightforward. From the remaining integrals, we assess typical $w$ (either from the cosine arguments, depending on $\omega$, or from algebraic factors), but ultimately, we need estimates for contributing times, so, to this end, we return to double integrals $37 \mathrm{a}$ and $38 \mathrm{a}$. Note at once that for given $w$ and $\omega$, typical $\tau$ are determined by the slope of the $\tau$-dependence of the phase, and by integration limits. The contribution from the end point

$$
\tau \approx \frac{T}{w}
$$

in Eqs. 37, 38a has the spread

$$
\delta \tau \sim \frac{2 \gamma^{2}}{\omega\left[1+\gamma^{2} \chi^{2} w(1-w)\right]},
$$

provided $\delta \tau \lesssim \tau$, which holds for sufficiently large $\omega$, or sufficiently small $w$. For the contribution from the end point $\tau=0$, typical $\tau$ and $\delta \tau$ are of the same order:

$$
\tau, \delta \tau \sim \frac{2 \gamma^{2}}{\omega\left[1+\gamma^{2} \chi^{2} w(1-w)\right]} .
$$

We will conduct this analysis up to the full spectral decomposition.

\section{A. Bethe-Heitler contribution}

It will be instructive to begin with figuring out typical contributing times for the simplest term $\frac{d I_{\mathrm{BH}}}{d \omega}(\gamma \chi)$. For visualization, let us first of all plot the integrand of Eq. (35), which is depicted in Fig. 7. More quantitative conclusions require scrutinizing the corresponding single integral (40). There, typical $w(1-w)$, i.e., effectively, $\min \{w, 1-w\}$, range from $\sim 1 / \gamma^{2} \chi^{2} \ll 1$ to $\sim 1$. According to Eq. (42), that corresponds to typical $\tau \sim \frac{2 \gamma^{2}}{\omega\left[1+\gamma^{2} \chi^{2} w(1-w)\right]}$ ranging from $l_{\chi}(\omega)$ to $l_{0}(\omega)$. Invoking relations $w=t_{2}^{\prime} / \tau, 1-w=-t_{1}^{\prime} / \tau$, that can be expressed in terms of $t_{1}^{\prime}$ and $t_{2}^{\prime}$ as

$$
\frac{\max \left\{\left|t_{1}^{\prime}\right|, t_{2}^{\prime}\right\}}{l_{0}(\omega)}+\frac{\min \left\{\left|t_{1}^{\prime}\right|, t_{2}^{\prime}\right\}}{l_{\chi}(\omega)} \sim 1 .
$$

The strong inequality between the contributing times, arising when $\left|t_{1}^{\prime}\right| \sim l_{0}(\omega) \gg t_{2}^{\prime} \sim l_{\chi}(\omega)$ or $\left|t_{1}^{\prime}\right| \sim$ $l_{\chi}(\omega) \ll t_{2}^{\prime} \sim l_{0}(\omega)$, reflects the fact that photons are intensely emitted along the initial or final electron direction. Among those, photons with coherence time $\left|t_{1}^{\prime}\right| \sim l_{0}(\omega)$ must be collinear to the initial electron (being intrajet), whereas $t_{2}^{\prime} \sim l_{\chi}(\omega)$ then represents the formation time for interjet photons (cf. Fig. 2), being significantly different from that for intrajet photon formation. The criterion of attributing the corresponding contribution to a jet is its independence of the electron scattering angle, whereas interjet radiation embodies all the dependence on this angle.



FIG. 7: Integrand of Eq. 35 at $\chi=30 \gamma^{-1}$ [case of single scattering, corresponding to $\left.\frac{d I_{\mathrm{BH}}}{d \omega}(\gamma \chi)\right]$. The extended populated regions correspond to photon emissions along one of the external electron lines.

The interference integral 39 may be treated in a similar manner [first determining $w$, and next the times from Eqs. (41) and (42)], but it requires different approximations in different spectral regions.

\section{B. High- $\omega$ domain. Intermediate electron contribution in Feynman gauge}

For the interference integral in Eq. (39), first consider the domain of high $\omega$, which is where the relatively simple intermediate line contribution must build up. In this limit, the cosine in Eq. 39 is rapidly oscillating. Integrals from oscillatory functions are dominated by points of stationary phase and end points of the integration interval [22]. In our case, there are no stationary phase points on the real axis, whereas the lower end point essentially does not contribute, because there $\cos \frac{\omega T}{2 \gamma^{2}}\left[\frac{1}{w}+\gamma^{2} \chi^{2}(1-w)\right]$ oscillates increasingly fast. Thus, the dominant contribution is brought by the upper end point alone, with the leading terms there being

$$
\begin{aligned}
& \int_{0}^{1} d w \cos \frac{\omega T}{2 \gamma^{2} w} \\
& -\int_{0}^{1} \frac{d w}{1+\frac{1}{\gamma^{2} \chi^{2}}-w} \cos \frac{\omega T}{2 \gamma^{2}}\left[\frac{1}{w}+\gamma^{2} \chi^{2}(1-w)\right]
\end{aligned}
$$

In the second integral (stemming from $\mathcal{I}_{e i}$ ), at $\gamma \chi \gg 1$ it is always legitimate replace in the phase $\frac{1}{w} \rightarrow 1$, since away from point $w=1$, this term plays minor role for any $\omega T$. Besides that, the lower integration end point may be replaced by $-\infty$, as long as $\frac{\omega T}{2 \gamma^{2}}$ is kept sizable. 
The result

$$
\begin{aligned}
& \int_{0}^{1} d w \cos \frac{\omega T}{2 \gamma^{2} w} \\
& -\int_{-\infty}^{1} \frac{d w}{1+\frac{1}{\gamma^{2} \chi^{2}}-w} \cos \frac{\omega T}{2 \gamma^{2}}\left[1+\gamma^{2} \chi^{2}(1-w)\right] \\
& =g\left(\frac{\omega T}{2 \gamma^{2}}\right)
\end{aligned}
$$

coincides with the intermediate electron line form factor (6).

It is worth noting that at $\omega \rightarrow \infty$, the leading $\mathcal{O}\left(\omega^{-1}\right)$ contributions from individual integrals in 43 mutually cancel, and the physical behavior $\sim \omega^{-2}$ is brought by the next-to-leading order contribution. In Sec. III] that property was attributed the vector character of electromagnetic radiation, via formation of the hollow cone angular distribution. Here, in the Feynman gauge, the cancellation engages the trajectory-independent part, represented by the first integral in (43) or (44).

From the lhs of Eq. 44, one infers that at $\frac{\omega T}{2 \gamma^{2}} \gg 1$, in both integrals typical $w=t_{2}^{\prime} / \tau$ are close to unity. That implies that $t_{2}^{\prime} \rightarrow \tau=t_{2}^{\prime}-t_{1}^{\prime}$, i.e., for $\mathcal{I}_{\text {ei }}$, the first correlation time $t_{1}^{\prime} \rightarrow-0$, tending to the first scattering point. From Eq. 41a we also see that $\tau \approx T / w \rightarrow T$, whence $t_{2}^{\prime} \rightarrow T$, i.e., it tends to the second scattering point, as is expectable physically. Finally, from the cosine factor of the second integral in Eq. (44), yielding

$$
1-w \sim \frac{2}{\omega T \chi^{2}}
$$

and from Eq. 41b), we get

$$
\delta \tau \sim l_{0}(\omega),
$$

which is natural from the collinear-collinear interference point of view. ${ }^{14}$ It is noteworthy that in spite of the dependence of one of the $\delta t$ 's on $\chi$, the resulting intermediate line spectral contribution (6b) is $\chi$-independent, insofar as the smallness of one of the time intervals, $\propto \frac{1}{\gamma^{2} \chi^{2}}$,

14 If one desires to estimate not only $\delta \tau$, but variations of each of the contributing times, as well, it is necessary to return to the original double-time representation (35), and linearize the argument of the sine in Eq. 35 about point $t_{1}^{\prime}=0, t_{2}^{\prime}=T$ :

$$
\sin \omega\left[\frac{\tau}{2 \gamma^{2}}-\frac{\chi^{2}}{2} \frac{t_{1}^{\prime} t_{2}^{\prime}}{T}\right] \simeq \sin \left[\frac{\omega}{2 \gamma^{2}}\left(T+\delta t_{2}^{\prime}\right)-\frac{\omega \chi^{2}}{2} \delta t_{1}^{\prime}\right] .
$$

This shows that the extents of the contributing time regions are unequal:

$$
\delta t_{1} \sim l_{\chi}(\omega) \ll \delta t_{2} \sim l_{0}(\omega) \ll T .
$$

For the $\mathcal{I}_{i e}$ part, vice versa, one would obtain

$$
\delta t_{1} \sim l_{0}(\omega) \gg \delta t_{2} \sim l_{\chi}(\omega) .
$$

Thus, at each end of the intermediate electron line, $l_{0}(\omega)$ and $l_{\chi}(\omega)$ enter on equal rights, although here, in contrast to $I_{\mathrm{BH}}$, they are adjacent not to one, but to different vertices. in the double time integral is exactly compensated by the prefactor containing one power of $\gamma^{2} \chi^{2}$ in the numerator. As for the phase, it is independent of $\chi$, granted that at $t_{1}^{\prime} \rightarrow 0,\left|\boldsymbol{r}\left(t_{2}^{\prime}\right)-\boldsymbol{r}\left(t_{1}^{\prime}\right)\right| \simeq v t_{2}^{\prime} \simeq v \tau$. Physically, the negligibility of the trajectory curvature, in spite of the trajectory bending to a substantial angle, is chained to the fact that at high $\omega$, this bending is felt only along a short distance.

\section{Low- $\omega$ domain. 'Radio' contribution and long time scales}

Next, let us turn to the domain of low $\omega$. It must be remembered that the intermediate line contribution extends there, as well. Approximation $w \approx 1$ for the second term of Eq. (43) remains valid even when $\omega \rightarrow 0$ - not because of the influence of the cosine factor (which varies slowly in the infrared limit), but due to the prefactor $\frac{1}{1+\gamma^{-2} \chi^{-2}-w}$ peaking near the end point. The only difference is that at $\omega T \chi^{2} / 2 \lesssim 1$, the lower end point in the second term cannot be replaced by $-\infty$, as in Eq. (44). But to cope with the latter impediment, and extend approximation (44) to the low- $\omega$ region (where its behavior will become logarithmic), it suffices just to subtract therefrom the corresponding lower end point contribution

$$
\begin{aligned}
& -\int_{-\infty}^{0} \frac{d w}{1+\frac{1}{\gamma^{2} \chi^{2}}-w} \cos \frac{\omega T}{2 \gamma^{2}}\left[1+\gamma^{2} \chi^{2}(1-w)\right] \\
& \approx-\int_{-\infty}^{0} \frac{d w}{1-w} \cos \frac{\omega T \chi^{2}}{2}(1-w)
\end{aligned}
$$

Combining 48 with the rest of the terms of 39 , in the low- $\omega$ limit one gets radio contribution in the form

$$
\begin{aligned}
& \int_{-\infty}^{0} \frac{d w}{1-w} \cos \frac{\omega T \chi^{2}}{2}(1-w) \\
& +\int_{0}^{\infty} d w\left(\frac{1}{w+\frac{1}{2 \gamma^{2} \chi^{2}}}-\frac{1}{w+\frac{1}{\gamma^{2} \chi^{2}}}\right) \cos \frac{\omega T}{2 \gamma^{2}}\left(\frac{1}{w}+\gamma^{2} \chi^{2}\right) \\
& +\int_{0}^{1} \frac{d w}{2-w} \cos \frac{\omega T \chi^{2}}{2}(1-w) .
\end{aligned}
$$

Here, in the second line we have neglected in the phase the small term linear in $w$, given typical $w \lesssim \gamma^{-2} \chi^{-2}$, and accordingly replaced the upper integration limit by infinity, whereas in the third line, on the contrary, term $\sim w^{-1}$ in the phase was neglected, since it affects the regular integrand only in a small vicinity of the origin. Thereby one separates in 49 the pure contribution from the end point $w=1$ :

$$
A_{2}\left(\frac{\omega T \chi^{2}}{2}\right)=\int_{-\infty}^{1} \frac{d w}{2-w} \cos \frac{\omega T \chi^{2}}{2}(1-w),
$$






FIG. 8: Antenna form factors in the case of coplanar electron scattering through two equal angles. $A_{1}$ (blue curve) is given by Eq. (51), and $A_{2}$ (red curve) by Eq. (50).

while the rest includes

$$
\begin{aligned}
A_{1}\left(\frac{\omega T \chi^{2}}{2}\right)=\int_{0}^{\infty} d w & \left(\frac{1}{w+\frac{1}{2 \gamma^{2} \chi^{2}}}-\frac{1}{w+\frac{1}{\gamma^{2} \chi^{2}}}\right) \\
& \times \cos \frac{\omega T}{2 \gamma^{2}}\left(\frac{1}{w}+\gamma^{2} \chi^{2}\right)
\end{aligned}
$$

and

$$
\int_{-\infty}^{0} d w\left(\frac{1}{1-w}-\frac{1}{2-w}\right) \cos \frac{\omega T \chi^{2}}{2}(1-w)
$$

A change of integration variable $\tilde{w}=-\frac{1}{\gamma^{2} \chi^{2} w}$ here reveals equality of contributions (51a) and (51b), hence, the result of integration in 49 amounts $2 A_{1}\left(\frac{\omega T \chi^{2}}{2}\right)+$ $A_{2}\left(\frac{\omega T \chi^{2}}{2}\right)$, in agreement with Eq. $13 \mathrm{~b}$. The behavior of form factors (50) and (51), which are now functions of a single variable, is illustrated in Fig. 8 .

For the evaluated radio part, again, it will be instructive first to plot the integrand of Eq. (32) in the $t_{1}^{\prime}, t_{2}^{\prime}$ plane (see Fig. 9), and with it in mind, analyze Eqs. (50), 51). From Eq. (51a), it follows that $w \sim 1 / \gamma^{2} \chi^{2} \ll 1$, wherewith Eq. 41) gives

$$
\tau \sim \frac{T}{w}=\gamma^{2} \chi^{2} T
$$

and

$$
\delta \tau \sim \frac{2 \gamma^{2}}{\omega\left(1+\gamma^{2} \chi^{2} w\right)} \sim l_{0}(\omega)
$$

[which at $\omega T \chi^{2} \sim 1$ is commensurable with 52 ]. In terms of absolute times, that implies

$$
t_{2}, t_{2}^{\prime} \sim w \tau \sim T, \quad\left|t_{1}\right| \approx \tau \sim \gamma^{2} \chi^{2} T \gg t_{2} .
$$

The long extent of one of those times, just like in the case of $I_{\mathrm{BH}}$, indicates that the photon is formed within the initial electron's proper field, and subsequently is stripped

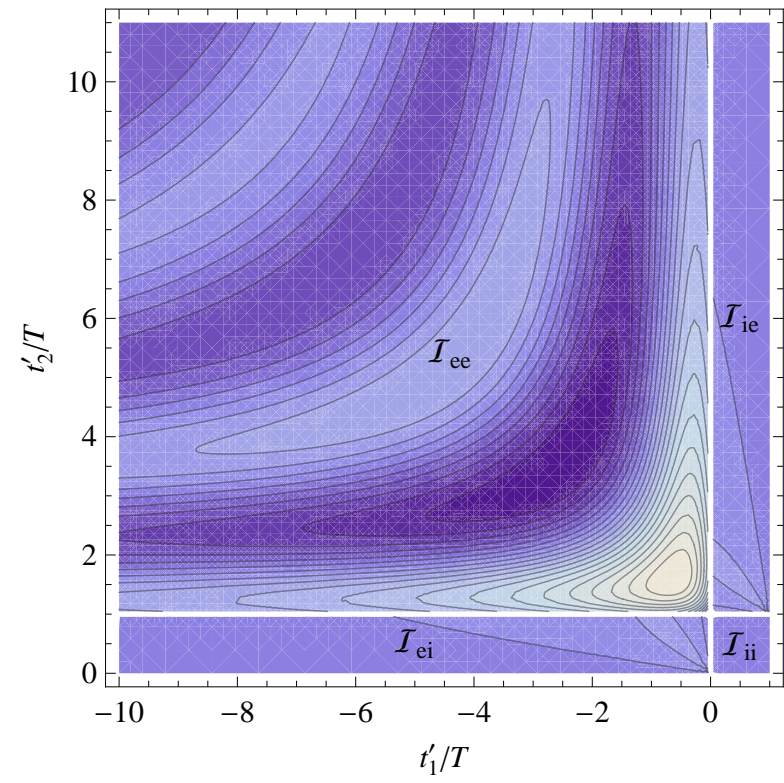

FIG. 9: Integrand of Eq. 32 at $\chi=30 \gamma^{-1}$ and $\frac{\omega T}{2 \gamma^{2}}=10^{-3}$. Most prominent is contribution $\mathcal{I}_{e e}$, similar to that of Fig. 7 but now corresponding to $\frac{d I_{\mathrm{BH}}}{d \omega}(2 \gamma \chi)$. Also noticeable are contributions from $\mathcal{I}_{e i}$ and $\mathcal{I}_{i e}$, corresponding to parts $(50)$ and $(51 \mathrm{~b})$ of the radio contribution, while part $\sqrt{51 \mathrm{a}}$ is too broad to be captured by this figure.

in the electron scattering region. Of course, there is also a cross-symmetric contribution, which has been taken into account implicitly, by symmetry.

On the other hand, in integrals (50) and (51b) typical $w$ are of the order unity, entailing

$$
\left|t_{1}\right| \sim t_{2} \sim T .
$$

That corresponds to the brightest spot in Fig. 9 , but it is directly related only with $A_{2}$, since in artificial integral (51b) all values of $w$ are unphysical (negative).

As we know from the preceding two sections, at $\omega T \sim$ $\chi^{-2}$, there arise spectral oscillations $\sim \frac{1}{\omega T} \sin \frac{\omega T \chi^{2}}{2}$, related with soft-collinear interference. Now we see that in integrals (51a) and (51b) they stem from small time ratios $w$ [in (51a), formally - from end point $w=\infty$, but presently, that implies just $\gamma^{-2} \chi^{-2} \ll w \ll 1$ ]. At the same time, contributing times for $A_{2}$ remain comparable with $T$. That is the physical reason why $A_{2}$ does not need to be supplemented by a form factor.

\section{Intermediate $\omega$ region: Decoherence and limits on ray optics}

With the increase of the photon frequency, terms $\propto w$ and $w^{-1}$ in the phase in Eq. 39 eventually become competing. That first happens in the spectral region $\omega T \sim \gamma / \chi$, and implies that bending of the electron trajectory during the photon formation process becomes es- 
sential (in accord with the notion of ray optics established in the previous section).

As was already mentioned, there is no stationary phase point on the real axis of $w$ (in contrast to the situation in Sec. IV), so, in order to find the saddle point, $w$ should be extended to the complex plane. But instead, it may suffice merely to note that typical contributing $w$ there are $\sim 1 / \gamma \chi \ll 1$. Simplifications are still possible then in the prefactors, which reduce to pure power laws. Yet, there is a nontrivial term $\frac{1}{1+\frac{1}{\gamma^{2} \chi^{2}}-w} \cos \frac{\omega T}{2 \gamma^{2}}\left[\frac{1}{w}+\gamma^{2} \chi^{2}(1-w)\right]$, which contributes not only at $w \sim 1 / \gamma \chi$, but also in vicinity of the end point $w=1$, where it blows up due to the smallness of the denominator. Those two different contributions in the same integral may be just added:

$$
\begin{aligned}
& \int_{0}^{1} d w \frac{1}{1+\frac{1}{\gamma^{2} \chi^{2}}-w} \cos \frac{\omega T}{2 \gamma^{2}}\left[\frac{1}{w}+\gamma^{2} \chi^{2}(1-w)\right] \\
& \simeq \int_{0}^{1} d w \frac{1}{1+\frac{1}{\gamma^{2} \chi^{2}}-w} \cos \frac{\omega T}{2 \gamma^{2}}\left[1+\gamma^{2} \chi^{2}(1-w)\right] \\
& +\int_{0}^{1} d w \cos \frac{\omega T}{2 \gamma^{2}}\left[\frac{1}{w}+\gamma^{2} \chi^{2}(1-w)\right] .
\end{aligned}
$$

In the first (upper end point) term, the lower limit may be replaced by $-\infty$, and along with term $\int_{0}^{1} d w \cos \frac{\omega T}{2 \gamma^{2} w}$, it constitutes the omni-present intermediate electron line contribution 444. The second (inner-point) term combines with the rest in Eq. (39) to give, under conditions $\gamma^{-2} \chi^{-2} \ll w \ll 1$,

$$
\begin{aligned}
& \int_{0}^{1} d w\left(\frac{1}{w+\frac{1}{2 \gamma^{2} \chi^{2}}}-\frac{1}{w+\frac{1}{\gamma^{2} \chi^{2}}}+\frac{1}{2-w}-1\right) \\
& \quad \times \cos \frac{\omega T}{2 \gamma^{2}}\left[\frac{1}{w}+\gamma^{2} \chi^{2}(1-w)\right] \\
& \simeq \frac{1}{2} \int_{0}^{\infty} d w\left(\frac{1}{\gamma^{2} \chi^{2} w^{2}}-1\right) \cos \frac{\omega T}{2 \gamma^{2}}\left[\frac{1}{w}+\gamma^{2} \chi^{2}(1-w)\right] \\
& =-\frac{2}{\gamma \chi} \sin \frac{\omega T \chi^{2}}{2} K_{1}\left(\frac{\omega T \chi}{\gamma}\right) .
\end{aligned}
$$

That is exactly asymptotics (15). Adding up intermediate line and $F_{\perp}$-modulated radio contributions, as in previous sections, ultimately recovers nondipole decomposition (22).

To estimate the relevant contributing times, let us note, again, that $\tau \rightarrow T / w$ [Eq. [41a)], wherefore $t_{2} \equiv w \tau / 2 \rightarrow T / 2, t_{2}^{\prime} \equiv w \tau \rightarrow T$, confirming that the second correlating time tends to the second scattering vertex, in the spirit of Fig. 5(b). As for $t_{1}$, estimate $w \sim 1 / \gamma \chi$ implies

$$
\tau \sim \gamma \chi T
$$

That is again consistent with the ray optic notions: longitudinal scale (57) equals the (fixed) transverse scale $T \chi$ divided by the natural jet collimation angle $\gamma^{-1}$. Hence,

$$
\tau,\left|t_{1}\right| \sim \gamma \chi T \gg t_{2} \sim T .
$$

Of course, there also exists a symmetric contribution $\left|t_{1}\right| \sim T \ll t_{2} \sim \gamma \chi T$, which had been taken into account implicitly by doubling $\mathcal{I}_{e i}$ and $\int_{0}^{1} d w \ldots$ in $\mathcal{I}_{e e}$.

Strong inequality (58) between the formation time scale for interfering photons is in accord with the causal origin of the factorization property: One of the two interfering components of the electromagnetic wave forms up long before or long after another (which forms fast), wherefore they are causally disconnected. At the same time, compared to the impact parameter approach, the notion of the ray of light along which the interference builds up is more uncertain here, because one of the correlating times is broadly distributed $(\delta \tau \sim \tau)$. Thus, in the present problem, even in the domain of its best applicability, the notion of ray optics is limited: The ray is well defined within the double scattering region, but cannot be extended down to the emission point. That makes the photon formation process in the present case akin to diffraction.

The exponential falloff here appears to be due to a superficially different reason - decoherence: Formally, the integrand in Eq. (56) assumes a saddle point on the imaginary axis of $w$, and it is the value of $e^{i \frac{\omega T}{2 \gamma^{2}}\left[\frac{1}{w}+\gamma^{2} \chi^{2}(1-w)\right]}$ in this point which converts to the exponentially decreasing factor. But physically, it is due to phase fluctuations (typical values of the $w$-dependent terms) $\frac{\omega T}{2 \gamma^{2} w}, \frac{\omega T \chi^{2}}{2} w \sim$ $\frac{\omega T \chi}{2 \gamma} \lesssim 1$, which grow with $\omega$, and progressively destroy the stability of the phase.

Although the mechanism of attenuation of low- $\omega$ spectral oscillations looks different in different frameworks, there is a noteworthy universal relation between the indeterminacies of the photon formation time and the transverse screening scale:

$$
\chi l_{\perp}^{-1}=\delta l_{\mathrm{f}}^{-1} .
$$

Here $\delta l_{\mathrm{f}}^{-1}$ is the indeterminacy of the reciprocal coherence length considered as a function of $\theta$ [Eq. (1)], or function of $w$

$$
l_{\mathrm{f}}^{-1}=\frac{\omega}{2 \gamma^{2}}\left[\frac{1}{w}+\gamma^{2} \chi^{2}(1-w)\right],
$$

with respect to typical indeterminacies $\delta \theta=2 \chi / \gamma$ or $\delta w \sim 1 / \gamma \chi$.

In those basic considerations, we could not bring out all the aspects of photon formation in the present process, so they may deserve additional investigation in the future.

\section{EXPERIMENTAL FEASIBILITY}

To accomplish the study of radiation at double electron scattering, it may also be expedient to discuss prospects for its experimental realization. Promising candidates for prompt deflection of relativistic particles to angles in excess of $\gamma^{-1}$ are thin crystals. There are several known crystal-assisted deflection mechanisms: channeling in a 
bent crystal [24, volume reflection in a bent crystal [25], and mirroring in a straight ultrathin "half-wavelength" crystal [26]. The acceptance to a stable channeling mode in practice may be insufficiently high (see, e.g., [27]), whereas for mirroring in a "half-wavelength" crystal, the relative spread in deflection angles must be sizable due to the impact parameter dependence. Volume reflection is not beset by such deficiencies, so we examine it in the first place.

Volume reflection develops over a length $\Delta z_{\mathrm{VR}} \sim R \theta_{c}$, and for the case of positively charged particles (for which it works somewhat better) leads to deflection to an angle $\chi \approx \frac{\pi}{2} \theta_{c}\left[28\right.$, where $\theta_{c}=\sqrt{2 V_{0} / E} \sim \sqrt{50 \mathrm{eV} / E}$ is the critical channeling angle. The intrinsic relative spread of the deflected beam is $\Delta \chi / \chi \sim 2 R_{c} / R$, where $R_{c}=$ $E /\left|F_{\max }\right|=0.2 \mathrm{~m} \frac{E}{\mathrm{GeV}}$ is the critical radius, and $R$ the crystal bending radius, which must be in excess of $4 R_{c}$.

Assuming positron energy $E=500 \mathrm{GeV}$, which can become available in the foreseeable future, such a positron can be deflected to an angle $\chi \approx 1.5 \theta_{c}=15 \mu \mathrm{rad}=15 \gamma^{-1}$ within a length $\Delta z_{\mathrm{VR}} \approx \frac{R}{R_{c}} 10 \mu \mathrm{m} \sim 0.1 \mathrm{~mm}$. The photon energy $\omega \sim \frac{1}{T \chi^{2}}=1 \frac{\mathrm{MeV} \mathrm{mm}}{T}$ will belong to soft gamma range $\omega \sim 1 \mathrm{MeV}$ provided the gap width amounts $T \sim 1$ $\mathrm{mm}$. As long as this is well in excess of $\Delta z_{\mathrm{VR}}$, the suggested setup should be feasible. At that, the additional angular spread due to incoherent multiple scattering on atomic nuclei in the crystal will be minor. An issue at such high an energy can be synchrotron radiation background from steering and focusing magnets, but it will be common for all the forward physics problems. Other mechanisms of crystal deflection demand more dedicated calculations.

Another option may be to utilize for deflection amorphous foils equipped by a position sensitivity system (charged particle tracking) enabling reconstruction of the electron trajectory and thereby selection of events of double hard scattering through prescribed angles. An issue therewith is that at momentum transfers $E \chi=$ $m_{e} \gamma \chi \gtrsim 20 m_{e} \sim 10 \mathrm{MeV}$, it may be important to take into account inner structure of atomic nuclei. If such a setup nonetheless proves feasible, the lower bound on the electron beam energy could be relaxed. Condition $\gamma \gtrsim \sqrt{\omega T / 2}$ (necessary for probing intermediate electron line resonances) with $\omega \sim 1 \mathrm{MeV}$ (to ensure transparency of both targets) and $T>0.2 \mathrm{~mm}$ implies $E=m_{e} \gamma>15$ $\mathrm{GeV}$. It has been actually tested in CERN, without electron tracking, at $E \sim 200 \mathrm{GeV}$ and $\omega \sim 1 \mathrm{GeV}$ [5]. As for condition $\chi^{-1} \sim \gamma / 30 \gtrsim \sqrt{\omega T / 2}$ necessary for testing radio resonances, it can be made compatible with CERN SPS energies $E \sim 200 \mathrm{GeV}$ for similar parameters $\omega \sim 1$ $\mathrm{MeV}$ and $T \sim 0.2 \mathrm{~mm}$.

If any kind of electron hard rescattering and observation of interference in the accompanying radiation will be realized, it would open prospects for experimental tests of coherence phenomena similar to those for quantum field theory jets. Let us remind that it is actually the coherence that distinguishes gauge field theory jets from purely random parton cascading [19]. At that, the notion of jets is usually associated with angular distributions, so it would be desirable as well to measure angular distributions of radiation like those in Fig. 2. Simultaneous measurement of photon energy and (small) emission angle is a challenge similar to that in gamma astronomy, which stimulates development of pixellated detector arrays 29$]$.

Finally, the electron deflection can be carried out by means of magnet deflectors in vacuum, but since magnet dimensions are always formidable, gap $T$ must be large, too, and correspondingly, the interesting radiation will not fall into gamma range. Experiments in optical region, including measurements of radiation angular distributions, had been undertaken some time ago 30. Under those conditions, though, one generally has to regard near-field effects (see, e.g., [18]).

\section{SUMMARY}

The principal prediction of the present paper is that when an electron is subjected to a double hard scattering through definite angles, the spectrum of the emitted radiation exhibits oscillations in two regions, reflecting manifestations of two coherence lengths: free $\left[l_{0}(\omega)\right]$ and electron scattering angle dependent $\left[l_{\chi}(\omega)\right]$.

The underlying reason for such an oscillatory behavior is the interplay of two categories of photons: those formed along straight parts of the electron's trajectory, with formation scale $l_{0}(\omega)$, and those emerging from relatively small vicinities of the trajectory break points, and forming at scale $l_{\chi}(\omega)$. Radiation of the first type is narrowly collimated along parent electron lines (intrajet, or collinear radiation), whereas that of the second type is broadly distributed in between the radiation jets (interjet radiation). Fainter angular distribution of the latter $\left(\sim \gamma^{-2} \chi^{-2}\right)$ is compensated by its wider occupied phase space $\left(\sim \gamma^{2} \chi^{2}\right)$, so in the angle-integral spectrum those contributions are comparable.

Spectral oscillations, persisting in spite of integration over all photon emission angles, arise when there are two interfering radiation components. At least one among them must be of collinear type, because by virtue of its natural narrow collimation properties, it can carry a welldefined phase. The second interfering component then must be emitted along the same direction. For the certainty of the phase, besides that, both components must have approximately equal impact parameters, i.e., effectively belong to the same ray in position space within the scattering region. One should then distinguish two kinds of interference geometries:

1. Interference between electromagnetic waves emitted from opposite ends of the intermediate segment of the electron's trajectory close to the direction of its velocity, and having small impact parameters [see Fig. 5(a)]. Both interfering waves here are 
collinear to the same electron line. This type of interference was discussed in 114, 17, 18.

2. Interference between electromagnetic waves, one of which is emitted from one of the external electron lines and keeps collinear to it, and another one (interjet), from the opposite vertex. Those waves propagate nearly parallel to the corresponding external electron line, at an impact parameter such that they pass through the opposite vertex [see Fig. 5(b)]. The photon formation length here amounts $l_{\chi}(\omega)$ - in spite of formation length for one of the waves being $l_{0}(\omega)$, the coherence length equals the smallest between the two.

The formal realization of the scale separation property is nondipole spectral decomposition (22). Therein, each term or factor depends on $\omega$ at its intrinsic scale, and contains appropriate approximations, but formally extends through the whole $\omega$ range. Interfering radiation from the intermediate electron line is associated with term $g\left(\omega T / 2 \gamma^{2}\right)$ given by Eq. (6). The rest of the terms are "radio" contributions factorizing into the quasiantenna [Eqs. (17), [18)] and the suppressing proper field form factors [Eq. [19]]. The latter form factors furnish the exponential damping of the soft spectral oscillations with the increase of $\omega$ due to localization of the interfering waves at a nonzero impact parameter $T \chi$, and due to decrease of the intrajet photon impact parameter distribution (on a scale given by the transverse coherence length), or, equivalently, due to fluctuations of the longitudinal coherence length [Eq. (59)]. Taken apart, soft and hard terms in the spectral density diverge at $\omega \rightarrow 0$ logarithmically, $g, r \sim \pm \ln \frac{1}{\omega}$ (cf. 31]), but their sum is finite.

It is likely that similar decomposition and factorization properties will prove relevant also in other problems involving continuous targets with sharp boundaries.

\section{Acknowledgements}

M.V.B. is grateful to A.V. Shchagin for bringing to his attention Ref. 17]. This work was supported in part by the National Academy of Sciences of Ukraine (Project No. CO-1-8/2016) and the Ministry of Education and Science of Ukraine (Project No. 0115U000473).

\section{Appendix A: Derivation of representation (32)}

Representation (2) in form of a double time integral

$$
\begin{array}{r}
\frac{d I}{d \omega}=\left(\frac{e \omega}{2 \pi}\right)^{2} \int d^{2} n \iint_{-\infty}^{\infty} d t_{1} d t_{2}\left[\boldsymbol{n} \times \boldsymbol{v}\left(t_{1}\right)\right] \cdot\left[\boldsymbol{n} \times \boldsymbol{v}\left(t_{2}\right)\right] \\
\times e^{i \omega\left(t_{1}-t_{2}\right)-i \boldsymbol{k} \cdot\left[\boldsymbol{r}\left(t_{1}\right)-\boldsymbol{r}\left(t_{2}\right)\right]}
\end{array}
$$

allows exact integration over radiation angles. To this end, $\int d^{2} n$ must be performed prior to integration over $t_{1}$ and $t_{2}$. It should be minded that time integrals in A1 are not absolutely convergent, so change of the integration order compared to Eq. (A1) must be done carefully. Problems arise in the limit $t_{2} \rightarrow t_{1}$, where the angular integral from the oscillatory exponential becomes singular. In particular, it may be necessary to treat the emerging singular function there as an improper one (a distribution).

Integration in A1 can be simplified by employing gauge invariance to reduce the power of $\boldsymbol{n}$ in the preexponential factor. Rewriting

$$
\left[\boldsymbol{n} \times \boldsymbol{v}\left(t_{1}\right)\right] \cdot\left[\boldsymbol{n} \times \boldsymbol{v}\left(t_{2}\right)\right]=v_{i}\left(t_{1}\right) v_{k}\left(t_{2}\right)\left(\delta_{i k}-n_{i} n_{k}\right),
$$

one can replace the photon polarization density matrix $\delta_{i k}-n_{i} n_{k}$ by that in the covariant (Feynman) gauge, proportional to the metric tensor $g_{\mu \nu}=\operatorname{diag}(1,-1,-1,-1)$ in Minkowski space-time, and not involving $\boldsymbol{n}$ :

$$
\begin{aligned}
d t_{1} d t_{2} v_{i}\left(t_{1}\right) v_{k}\left(t_{2}\right)\left(\delta_{i k}-n_{i} n_{k}\right) & \rightarrow-d s_{1} d s_{2} u_{\mu}\left(t_{1}\right) u_{\nu}\left(t_{2}\right) g_{\mu \nu} \\
& =d t_{1} d t_{2}\left[\boldsymbol{v}\left(t_{1}\right) \cdot \boldsymbol{v}\left(t_{2}\right)-1\right]
\end{aligned}
$$

where $u_{\mu}=d r_{\mu} / d s=\gamma(1, \boldsymbol{v}), d s=\sqrt{d t^{2}-d \boldsymbol{r}^{2}}=d t / \gamma$. The validity of form (A3) can equally well be justified via integration by parts in the second term of $\mathrm{A} 2$ :

$$
\begin{gathered}
\int_{-\infty}^{\infty} d t \boldsymbol{n} \cdot \boldsymbol{v}(t) e^{i\{\omega t-\boldsymbol{k} \cdot \boldsymbol{r}(t)\}}=\frac{i}{\omega} \int_{-\infty}^{\infty} d t e^{i \omega t} \frac{\partial}{\partial t} e^{-i \boldsymbol{k} \cdot \boldsymbol{r}(t)} \\
=-\frac{i}{\omega} \int_{-\infty}^{\infty} d t e^{-i \boldsymbol{k} \cdot \boldsymbol{r}(t)} \frac{\partial}{\partial t} e^{i \omega t}=\int_{-\infty}^{\infty} d t e^{i \omega t-i \boldsymbol{k} \cdot \boldsymbol{r}(t)}
\end{gathered}
$$

for each of the times $t_{1}, t_{2}$. The change of the gauge as a result of integration by parts is the fundamental property of electrodynamics 14].

Inserting (A3) to A1] and making simplifications pertinent to the ultrarelativistic limit, leads to 15

$$
\begin{aligned}
& \frac{d I}{d \omega}=-\left(\frac{e \omega}{2 \pi}\right)^{2} \int d^{2} n \iint_{-\infty}^{\infty} d t_{1} d t_{2} \\
& \times\left\{\gamma^{-2}+\frac{1}{2}\left[\boldsymbol{v}\left(t_{2}\right)-\boldsymbol{v}\left(t_{1}\right)\right]^{2}\right\} e^{i \omega\left(t_{1}-t_{2}\right)-i \boldsymbol{k} \cdot\left[\boldsymbol{r}\left(t_{1}\right)-\boldsymbol{r}\left(t_{2}\right)\right]} .
\end{aligned}
$$

Here it was presumed that $\boldsymbol{v}^{2}\left(t_{1}\right)=\boldsymbol{v}^{2}\left(t_{2}\right)=1-\gamma^{-2}$ is time independent (otherwise $\gamma^{-2}$ must be replaced by $\left.\frac{1}{2}\left[\gamma^{-2}\left(t_{1}\right)+\gamma^{-2}\left(t_{2}\right)\right]\right)$.

Next, we employ the symmetry between $t_{1}$ and $t_{2}$ to write $\iint_{-\infty}^{\infty} d t_{1} d t_{2} \ldots=2 \mathfrak{R e} \int_{-\infty}^{\infty} d t_{2} \int_{-\infty}^{t_{2}} d t_{1} \ldots$, and note that integral

$$
\int d^{2} n e^{i \boldsymbol{k} \cdot\left[\boldsymbol{r}\left(t_{2}\right)-\boldsymbol{r}\left(t_{1}\right)\right]}=\pi \int_{0}^{\infty} d n_{\perp}^{2} e^{i \omega\left(1-n_{\perp}^{2} / 2\right)\left|\boldsymbol{r}\left(t_{2}\right)-\boldsymbol{r}\left(t_{1}\right)\right|}
$$

will converge absolutely provided we replace $\left|\boldsymbol{r}\left(t_{2}\right)-\boldsymbol{r}\left(t_{1}\right)\right| \rightarrow\left|\boldsymbol{r}\left(t_{2}\right)-\boldsymbol{r}\left(t_{1}\right)\right|-i \epsilon$, where $\epsilon \rightarrow+0$. The integration then gives 32 ]

$$
\begin{aligned}
\frac{d I}{d \omega}=-\omega \frac{e^{2}}{\pi} & \int_{-\infty}^{\infty} d t_{2} \int_{-\infty}^{t_{2}} d t_{1}\left\{\gamma^{-2}+\frac{1}{2}\left[\boldsymbol{v}\left(t_{2}\right)-\boldsymbol{v}\left(t_{1}\right)\right]^{2}\right\} \\
& \times \mathfrak{I m} \frac{1}{t_{2}-t_{1}-i \epsilon} e^{-i \omega\left[t_{2}-t_{1}-\left|\boldsymbol{r}\left(t_{2}\right)-\boldsymbol{r}\left(t_{1}\right)\right|\right]},
\end{aligned}
$$




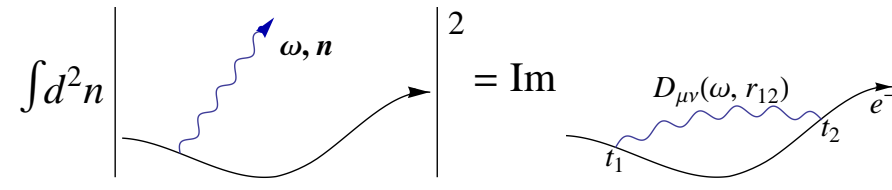

FIG. 10: Graphical illustration of Eq. A7).

where we replaced in the preexponential factor $\left|\boldsymbol{r}\left(t_{2}\right)-\boldsymbol{r}\left(t_{1}\right)\right| \approx t_{2}-t_{1}$, while in the phase factor such a replacement is generally not justified.

The meaning of formula $\mathrm{A} 6$ becomes obvious when written covariantly as

$$
\begin{aligned}
\frac{d I}{d \omega}= & \omega \frac{e^{2}}{\pi} \int_{-\infty}^{\infty} d s_{2} \int_{-\infty}^{s_{2}} d s_{1} u_{\mu}\left(t_{1}\right) u_{\nu}\left(t_{2}\right) \\
& \times \mathfrak{I m} e^{-i \omega\left(t_{2}-t_{1}\right)} D_{\mu \nu}\left(\omega,\left|\boldsymbol{r}\left(t_{2}\right)-\boldsymbol{r}\left(t_{1}\right)\right|\right),
\end{aligned}
$$

where

$$
D_{\mu \nu}(\omega, r)=-\frac{g_{\mu \nu}}{r-i \epsilon} e^{i \omega r}
$$

is the photon propagator in Feynman gauge and frequency-position representation 9] (appropriately regularized at $r=0$, which would have no effect in quantum electrodynamics, but is essential in classical). Equation (A7) expresses nothing but the unitarity relation (cf., e.g., 9 ) between the angle-integral real photon emission probability $\frac{1}{\hbar \omega} \frac{d I}{d \omega}$ and the imaginary part of a virtual photon propagator inserted between two points on the electron trajectory - as is graphically illustrated in Fig. 10 Notation (A7) is gauge invariant, holding in any gauge for the photon propagator, but the use of Feynman gauge is arguably the simplest.

The effect of infinitesimal term $-i \epsilon$ in the denominator of $D_{\mu \nu}$ is that

$$
\begin{aligned}
& -\mathfrak{I m} \frac{1}{t_{2}-t_{1}-i \epsilon} e^{-i \omega\left[t_{2}-t_{1}-\left|\boldsymbol{r}\left(t_{2}\right)-\boldsymbol{r}\left(t_{1}\right)\right|\right]} \\
& \underset{\epsilon \rightarrow+0}{\rightarrow} \frac{\sin \omega\left[t_{2}-t_{1}-\left|\boldsymbol{r}\left(t_{2}\right)-\boldsymbol{r}\left(t_{1}\right)\right|\right]}{t_{2}-t_{1}}-\pi \delta\left(t_{2}-t_{1}\right) .(
\end{aligned}
$$

Here, since the singularity point of the emerging delta function falls onto the integration domain edge in Eq. (A6), due to the symmetry between $t_{1}$ and $t_{2}$, the contribution from the delta function must be regarded as halved. Owing to the last term, the radiation spectrum vanishes for a uniform and rectilinear electron motion.

In practice, it may be convenient to replace the delta function (the instantaneous term) in $\mathrm{A} 8 \mathrm{~b}$ by a regular function producing an identical effect. Customarily, it is written as

$$
\begin{aligned}
\frac{d I}{d \omega}=\omega \frac{e^{2}}{\pi} \int_{0}^{\infty} \frac{d \tau}{\tau} \int_{-\infty}^{\infty} d t_{2}( & \left\{\gamma^{-2}+\frac{1}{2}\left[\boldsymbol{v}\left(t_{2}\right)-\boldsymbol{v}\left(t_{2}-\tau\right)\right]^{2}\right\} \\
\times \sin \omega[ & \left.\tau-\left|\boldsymbol{r}\left(t_{2}\right)-\boldsymbol{r}\left(t_{2}-\tau\right)\right|\right] \\
& \left.-\gamma^{-2} \sin \mathcal{K} \tau\right)
\end{aligned}
$$

with $\mathcal{K} \rightarrow \infty$, or, since $\int_{0}^{\infty} \frac{d \tau}{\tau} \sin \mathcal{K} \tau=\frac{\pi}{2}$ is actually $\mathcal{K}$-independent, in form (32). The advantage of the latter form is that for a uniformly and rectilinearly moving charge, the integrand rather than only the whole integral turns to zero. (Yet, since the integrand becomes decreasing as $\left|t_{2}\right| \rightarrow \infty$, it affords one to interchange the order of integrations.) Equation (32) is the subtracted Blankenbecler-Drell formula 33, which was derived here without introducing the "vacuum" term by hand.

It is also worth noting that representation $(\mathrm{A9})$ with $\mathcal{K}=2 \omega$ can be obtained directly if one integrates not only over typical small photon emission angles, but over the full solid angle [15]. Then, $\sin 2 \omega \tau$ is associated with "backward" radiation, which may be physically negligible, but is suitable for regularizing the integral.
[1] R. Blankenbecler, Phys. Rev. D 55, 190 (1997).

[2] B.G. Zakharov, Pis'ma Zh. Eksp. Teor. Fiz. 64, 737 (1996) [JETP Lett. 64, 781 (1996)]; Yad. Fiz. 61, 924 (1998) [Phys. At. Nucl. 62, 1008 (1998)].

[3] V.N. Baier and V.M. Katkov, Phys. Rev. D 60, 076001 (1999).

[4] M.V. Bondarenco and N.F. Shul'ga, Phys. Rev. D 90, 116007 (2014); 95, 019901(E) (2017).

[5] K.K. Andersen et al., Phys. Lett. B 732, 309 (2014).

[6] L.D. Landau and I.Ya. Pomeranchuk, Dokl. Akad. Nauk. SSSR 92, 535 (1953); ibid., 92, 735 (1953).

[7] A.B. Migdal, Phys. Rev. 103, 1811 (1956).

[8] V.M. Galitsky and I.I. Gurevich, Nuov. Cim. 32, 396 (1964).
[9] V.B. Berestetskii, E.M. Lifshitz, L.P. Pitaevskii, Quantum Electrodynamics, Oxford: Pergamon-Press, 1982.

[10] S. Klein, Rev. Mod. Phys. 71, 1501 (1999).

[11] E. Purcell. Electricity and Magnetism, New York: McGraw Hill, 1965.

[12] E.L. Feynberg, Zh. Eksp. Teor. Fiz. 50, 202 (1966).

[13] A.I. Akhiezer, V.B. Berestetskii. Quantum Electrodynamics, Moscow: Nauka, 1981 (in Russian).

[14] J.D. Jackson. Classical Electrodynamics, 3rd ed., New York: Wiley, 1998.

[15] A.I. Akhiezer and N.F. Shul'ga,Usp. Fiz. Nauk 137, 561 (1982) [Sov. Phys. Usp. 25, 541 (1982)]; A.I. Akhiezer, N.F. Shul'ga, High Energy Electrodynamics in Matter, Amsterdam: Gordon and Breach, 1996. 
[16] S.P. Fomin, N.F. Shul'ga, and S.N. Shul'ga, Yad. Fiz. 66, 421 (2003) [Phys. At. Nucl. 66, 394 (2003)].

[17] O.V. Chubar and N.V. Smolyakov, J. Optics (Paris) 24, 117 (1993); R.A. Bosch, Il Nuovo Cim. 20, 483 (1998).

[18] G. Geloni et al., NIM A 605, 409 (2009), 607, 470 (2009).

[19] Yu.L. Dokshitzer, V.A. Khoze, A.H. Mueller, and S.I. Troyan, Rev. Mod. Phys. 60, 373 (1988); Basics of Perturbative QCD, Paris: Editions Fontieres, 1991.

[20] M. Abramowitz, I.A. Stegun. Handbook of Mathematical Functions. New York: Dover, 1972.

[21] S. Coleman and R.E. Norton, Nuov. Cim. 38, 438 (1965).

[22] F.W.J. Olver. Asymptotics and Special Functions, 2nd ed., Taylor \& Francis, 1997.

[23] S. Weinberg. The Quantum Theory of Fields (Vol. 1), Cambridge: Univ. Press, 1996.

J. Collins, Foundations of Perturbative QCD, Cambridge: Univ. Press, 2011.

[24] E.N. Tsyganov. Fermilab TM-682, 1976 (preprint); V.B.S. Strokov et al., J. Phys. Soc. Jpn. 76, 064007 (2007); V.M. Biryukov, Y.A. Chesnokov, V.I. Kotov, Crystal Channeling and Its Application at High-Energy Accelerators, Berlin: Springer, 2010.
[25] A.M. Taratin and S.A. Vorobiev. NIM B 26, 512 (1987); W. Scandale et al., Phys. Rev. A 79, 012903 (2009); U. Wienands et al., Phys. Rev. Lett. 114, 074801 (2015).

[26] E.N. Tsyganov and A.M. Taratin, NIM A 363, 511 (1995); W. Scandale et al., Phys. Lett. B 734, 1 (2014).

[27] E. Bagli et al., Eur. Phys. J. C 74, 2740 (2014).

[28] M.V. Bondarenco, Phys. Rev. A 82, 042902 (2010); Il Nuov. Cim. C 34, 381 (2011); Phys. Rev. ST Accel. Beams 15, 032802 (2012).

[29] C. Labanti et al., Astron. Astrophys. 411, L149 (2003).

[30] M.M. Nikitin, A.F. Medvedev, M.B. Moiseev, and V.Ya. Epp. Zh.E.T.F. 79, 763 (1980) [Sov. Phys. JETP 52, 388 (1980)].

[31] I.I. Gol'dman, Zh.E.T.F. 38, 1866 (1960) [Sov. Phys. JETP 11, 1341 (1960)].

[32] V.N. Baier, V.M. Katkov, V.M. Strakhovenko. Electromagnetic Processes at High Energies in Oriented Crystals, Singapore: World Scientific, 1998.

[33] R. Blankenbecler and S.D. Drell, Phys. Rev. D 53, 6265 (1996). 